ANL/ HEP-7211

NAL/THY 45

YALE-3075/4

Mar. 1972

CORRELATIONS IN HIGH-ENERGY PRODUCTION PROCESSES $^{\dagger}$

EDMOND L. BERGER

High Energy Physics Division

Argonne National Laboratory

Argonne, Illinois $60439^{\circ}$

M. JACOB

National Accelerator Laboratory

Batavia, Illinois 60510

and

R. SLANSKY $* *$

Physics Department

Yale University

New Haven, Connecticut 06520

\footnotetext{
$\mp$ Work performed under the auspices of the U. S. Atomic Energy Commission.

$*$

On leave from CERN, Geneva, Switzerland.

* Supported in part by AEC contract AT(11-1) 3075.
} 


\begin{abstract}
$\underline{\text { ABSTRACT }}$
Rapidity distributions for two particle inclusive reactions are calculated from the Nova model which has been successful at reproducing the single particle distributions. Particular attention is given to the $2 \pi^{-}$correlations in $\pi^{ \pm} p$ and pp collisions for which data are available. Good agreement with data provides further evidence for a strong clustering of secondaries in particle production at present machine energies, a feature inferred previously from the analysis of single particle distributions. We stress the importance of measur ing the energy dependence of $\langle n(n-1)\rangle$, the quantity which normalizes the two particle distribution. A number of other features of correlation experiments are discussed. General properties of particle production are emphasized in reviewing the Nova model.
\end{abstract}


I. Introduction.

Inelastic processes represent approximately $80 \%$ of the total strong interaction cross section at high energy. However, the large number of potentially important kinematic variables necessary for a complete description of these processes has hampered progress toward their understanding. Finding good ways to display available data is a fascinating challenge. Advent of the inclusive approach ${ }^{(1-4)}$ has simplified matters somewhat. Single particle inclusive cross sections may be described by only three kinematic variables, but even here, a particular choice of a given set must be made. These complications make it hard to approach particle production without some prejudices, even though one would wish to remain open to all suggestions obtainable from an unbiased contemplation of data. Fortunately, a motivated interest in one or a few variables may be rewarded by some insight and suggest further investigations. This has certainly been the case with the single-particle distributions, where rapidity y and transverse momentum squared $\mathrm{p}_{\mathrm{T}}{ }^{2}$ distributions of one specific secondary are measured ${ }^{5}$, with everything else summed or averaged over. Single-particle distributions are quite accessible experimentally, and they appear to provide tests of asymptotically expected scaling properties. $(1,2)$ Asymptotic limits and the approach to scaling have both been confronted in Regge theor $\mathrm{y}^{6}$, and 
its associated phenomenology. ${ }^{7}$ The next phase of inclusive phenomenology is a study of two-particle inclusive distributions and possible correlations among produced secondaries.

A number of features of single-particle inclusive spectra encourage the attempt to identify some dominant production mechanisms, which could serve as a basis for analyzing reactions in more detail. 4 Focusing on specific production mechanisms could be useful for the complicated problem of two-particle correlations. Although such an approach risks loss of generality, it is clear that detailed calculations are necessary if progress is to be made. General arguments based on the use of sum rules ${ }^{8}$ have been developed, but their implications for data are as yet very weak, in particular for slow pions in the center of mass, where the cross section is largest. Energy-momentum conservation alone imposes little constraint on shapes of single and two-particle distributions. Because data on two-particle distributions are accumulating rapidly; ${ }^{(9-12)}$ is should be extremely useful to have explicit model predicitions from which to view the data.

Asymptotic predictions for two-particle correlations have already been studied in the framework of Regge, dual, and diffraction models. $(13-16)$ In these models correlations are expected to decrease with increasing rapidity difference $(\Delta y \geq 2)$, but some models require a very large center of mass energy before detailed study is possible. 
This is especially true of models which postulate relatively weak correlations among secondaries originating from different clusters along a multi-exchange chain. One may also use the Feynman gas analogy $^{1,16}$ applicable when the rapidity distribution has become asymptotically uni form. In the gas, clustering fluctuations may occur which will lead to short-range correlations, and a definite correlation length in $\Delta y$. A problem in applying this analogy to present machine energies is that in several interesting cases the number of "gas molecules" involved, on the average; equals one to two (See Table I). In spite of the value of asymptotic approaches and formal analogies, presently available data must also be studied along different lines.

A glance at the single-pion distributions already provides some model independent expectations for two-pion distributions. Figure 1 shows the $\pi^{-}$distributions in $18.5 \mathrm{GeV} / \mathrm{c} \pi^{+} \mathrm{p}$ and $\pi^{-} \mathrm{p}$ collisions. 9 The shape in rapidity is narrow and Gaussian-like. In addition a strong leading particle effect is present in the $\pi^{-} p$ reaction. Whatever the reason for the Gaussian-like shape, the narrowness of the rapidity distribution indicates that the two-particle distribution will have its maximum when both rapidities are small, and hence, when the rapidity difference is small. The experimental evidence is for a prominent maximum at zero rapidity difference in the two $\pi^{-}$system. $(10,11)$ The double distribution $(9,12) \mathrm{d}^{2} d \mathrm{dy}_{1} \mathrm{dy} \mathrm{y}_{2}$ peaks near $\mathrm{y}_{1}=0$ for any fixed $y_{2}$ with the maximum shifting only lightly as a function of $y_{2}$. 
Thus $d^{2} \sigma / d y_{1} d y_{2}$ displays much more information ${ }^{(9,12)}$ than $d \sigma / d(\Delta y)$ which shows only a deceptive maximum near $\Delta y=0$. A very interesting problem is to account for the narrowness of both the one- and twopion distributions.

The observed strength and distribution of the leading particle effects in $\pi p$ and $p p$ collisions indeed provide some understanding why the single-pion rapidity distributions are so narrow. (17, 18) In manyevents, one of the initial particles is quasielastically scattered and carries off a sizable fraction of the energy. (19) The energy left to be shared among the remaining secondaries is small enough that produced pions tend to cluster at small rapidities. This is the weak inelasticity property which dominates production processes at present machine energy. (20) It finds a rationale in several theor etical approaches. Moreover, the rapid scaling of the invariant pion distributions at intermediate values of $|x|$ strongly suggests that pions which reach these intermediate values of $|x|$ come from energy independent. production of low mass clusters. Very little more than this is needed for understanding single-particle distributions and, as discussed here, the correlation data. Since the mass of a cluster which gives two $\pi^{-}$must be relatively large, one would expect $d^{2} \sigma / d y_{1} d y_{2}$, to be narrower than $d \sigma / d y$. It is possible to construct a rather general model based on these features because the distribution of secondaries from each cluster is quite independent of reaction and particle-type, 
a feature suggested by the nearly universal $\mathrm{p}_{\mathrm{T}}{ }^{2}$ distributions observed experimentally. We emphasize that the general behavior of the correlations can be accounted for by these experimental featur es of particle production. With only a few more assumptions they are easily incorporated into a specific model such as the Nova model.

The object of this paper is to show that a model which incorporates the key features already mentioned and thus reproduces the singleparticle distributions very well, ${ }^{(21)}$ can also account for the twoparticle distributions. $(12,22)$ The shapes are basically those expected from the observed small inelasticity, the interpretation of the rapid scaling at intermediate $|x|$ found in the model, and the general behavior of the $\mathrm{p}_{\mathrm{T}}{ }^{2}$ distributions. ${ }^{(3,4)}$ The very general clustering effect already inferred from the analysis of single distribution is enough to explain the two-particle correlation results now available. Two-particle data therefore provide further tests for some ideas which were less well probed by the single-particle distributions, expecially some of the details of particle production, but do not suggest specific additional effects.

In this paper, we also emphasize the great value of obtaining experimental information on the energy dependence of a quantity which actually normalizes the two-particle distribution, namely $<\mathrm{n}(\mathrm{n}-1)>$. The analogous quantity in the single-particle case is the mean multiplicity, $\langle n>$, of the ob served particle type. 


$$
\begin{aligned}
& \langle n(n-1)\rangle \sigma_{\text {ince }}=\int \frac{d^{2} \sigma}{d y_{1} d y_{2}} d y_{1} d y_{2} \\
& \langle n\rangle \sigma_{\text {incl }}=\int \frac{d \sigma}{d y} d y
\end{aligned}
$$

One of the most revealing and sensitive quantities which model calculations must account for is the energy dependence of the ratio, $\langle\mathrm{n}(\mathrm{n}-1)\rangle \mid\langle\mathrm{n}\rangle^{2}$ for secondaries of a specific kind.

Calculations of $\pi^{-} \pi^{-}$distributions are described in Sec. II. Strong correlations, already suggested by the single-particle distributions and observed in analyses of exclusive channels, are realized by associating most of the time the two observed pions with the excita tions of either the beam or target particle into a resonance-like state (Nova) which decays subsequently by pion emission. The Nova mode1 ${ }^{21}$ is reviewed briefly in the course of setting up the two-particle inclus ive calculation. Specific predictions of the model agree well with available correlation data. Calculations of the $\pi^{+} \mathrm{p}\left(\pi^{-}, \pi^{-}\right)$distribution ${ }^{(24)}$ at $18.5 \mathrm{GeV} / \mathrm{c}$ which have alr eady been reported elsewhere, ${ }^{(22)}$ are discussed in more detail. Those results showed deviations from data for large differences of rapidity where cross sections are small. This discrepancy suggests an obvious double excitation correction to the dominant single-excitation picture. We find that data are well accounted for if $70 \%$ of the inelastic cross section is associated with 
single excitation, and the remainder comes from events in which both beam and target particles form clusters. The $7 / 3$ ratio was also found to lead to a good representation of $\mathrm{pp}\left(\pi^{-}, \pi^{-}\right)$. The ratio of single to double excitation is tested by the reaction, $\pi^{-} \mathrm{p}\left(\pi^{-}, \pi^{-}\right)$where $\mathrm{d}^{2} \sigma / d y_{1} d y_{2}$ is extremely sensitive to the presence of leading $\pi^{-}$'s. Results are presented here for $\mathrm{pp}\left(\pi^{-}, \pi^{-}\right)^{(12)}$ and for $\pi^{ \pm} \mathrm{p}\left(\pi^{-}, \pi^{-}\right)$. $^{(9)}$

Normalizing to $\sigma_{\text {inel }}$, we find that our model reproduces correctly the normalization of the two-particle distributions. Results of our calculations are compared with data in Fig. 1, 3, 4, 5, and 6 . The agreement with experiment provides evidence that the pion secondaries are indeed strongly correlated at present machine energies, but in a well understood way. Correlations from final state interactions are minimized by the choice of $\pi^{-} \pi^{-}$, for which no strong resonance effects should mask correlations resulting from the production mechanism. It is therefore fortunate to see that the charge configuration which is most easily amenable for experimental study turns out to be of particular theoretical interes.

As reported previously, ${ }^{(12)}$ for $\pi^{-}$production in pp collisions our approach reproduces correctly the values and energy dependence of $\langle n\rangle,\langle n(n-1)\rangle$ (and $f_{2}=\langle n(n-1)\rangle-\langle n\rangle^{2}$ ) over the energy range 10-30 GeV/c. Further exploration of production mechanisms would follow from a measurement of the ratio $\langle\mathrm{n}(\mathrm{n}-1)\rangle /\langle\mathrm{n}\rangle^{2}$ at high energies, since models differ widely on the energy dependence of this quantity.

* In defining such a ratio, we arbitrarily consider most heavy novas as due to single excitation. In this case there is neither a neat kinematical nor a clear dynamical separation between single and double excitation. 
The success of our model in the $10-30 \mathrm{GeV} / \mathrm{c}$ range does not imply solid predictions at NAL or ISR energies. High mass excitations become more important at these very high energies, but the nova production cross section and decay multiplicity are not determined uniquely by the slow increase of $\langle n\rangle$ with energy, the only important energy dependent input to our model. Predictions for the singleparticle distributions are safer, since their normalization is determined by the already established slow increase of multiplicity. (25) The normalization of $d^{2} \sigma / d y_{1} d y_{2}$ increases in proportion to $\sqrt{s}$ in fragmentation models. Predictions for NAL energies are given. These points are discussed in Sec. III. Moments higher than $\langle n(n-1)\rangle$ would, of course, also be interesting in the NAL ISR energy domain. Cosmic ray results provide some hints of what to expect. $(26,27)$

In Sec. IV we close with a few general remarks and predictions about other easily accessible meson correlations. Calculations are presented for $\mathrm{K}^{+} \mathrm{p}\left(\mathrm{K}^{0}, \pi^{-}\right)$where one also has an exotic system.

II. Two-Particle Inclusive Distributions in the Nova Model.

In this section, we discuss our model calculation of two-particle inclusive distributions at present machine momenta $(10$ to $30 \mathrm{GeV} / \mathrm{c})$. For definiteness, we examine the specific reactions $\pi^{ \pm} \mathrm{p}\left(\pi^{-}, \pi^{-}\right)$, but most results are carried over easily to other processes. More details are given also of previously reported results ${ }^{(12)}$ on $\mathrm{pp}\left(\pi^{-}, \pi^{-}\right)$. The two 
final pions are labeled with indices 1 and 2. The two-particle inclusive distribution is a function of 6 kinematic variables, which may be taken to be the two longitudinal rapidities of the final pions, $y_{1}$ and $y_{2}$, in the center-of-mass frame; two transverse momenta $\left|\mathrm{p}_{\mathrm{T} 1}\right|$ and $\left|\mathrm{p}_{\mathrm{T} 2}\right|$; the angle $\phi$ between the two transverse vectors; and the center-of-mass energy $\sqrt{s}$. In this Section we focus on $y_{1}$ and $y_{2}$ dependence, integrating explicitly over $\mathrm{p}_{\mathrm{T} 1}^{2}$ and $\mathrm{p}_{\mathrm{T} 2}^{2}$. Thus, the quantity analyzed here is $\mathrm{d}^{2} \mathrm{\sigma} / \mathrm{dy}_{1} \mathrm{dy}_{2}$. Remarks are made in Section IV concerning dependence on $\mathrm{p}_{\mathrm{T} 1}, \mathrm{p}_{\mathrm{T} 2}$, and $\phi$. Energy dependence is discussed in Section III.

A. Review of the Model

As emphasized in the Introduction, the Nova model is a phenomenological approach which embodies three features abstracted from data on single-particle inclusive spectra. ${ }^{(3,4)}$ We examine each of these features in succession.

1. High energy collisions are termed "weakly inelastic" in that one of the incident particles often retains a large fraction of its center-of-mass incident energy. This fact is manifest in various ways. For example, in $p p(p)$ the final proton's Iongitudinal momentum spectrum is observed to be flat, $(19,28)$ or even to be slightly peaked towards the kinematic limit $\left|\mathrm{p}_{L}\right|=\mathrm{p}_{\mathrm{L}}, \max$. In pion production processes, ${ }^{(9,18)}$ an excess of $\pi^{-}$in $\pi^{-} \mathrm{p}\left(\pi^{-}\right)$is observed at $y \geq 2$. This effect is seen in data displayed in Fig. 1, but shows up more dramatically when data are plotted versus $\mathrm{x}$, or versus $\mathrm{y}$ for 
small $\mathrm{p}_{\mathrm{T}}$ intervals. ${ }^{(18)}$ The excess is associated with events in which the $\pi^{-}$is quasi-elastically scattered, recoiling from an excited proton-like system ("cluster" or "nova"). Small inelasticity already implies some correlation among secondaries, ${ }^{(20)}$ because secondaries decaying from the excited cluster share a fraction of center-of-mass energy which is substantially less than that which would be awarded by energy-momentum conservation alone.

To represent small inelasticity in as obvious a fashion as possible, we use single cluster excitation diagrams sketched in Fig. 2(a) and (b). In addition, our fits $(12,22)$ to $d^{2} \sigma / d y_{1} d y_{2}$ demonstrate the need for contributions of the type shown in Fig. 2(c), in which both beam and target are excited in a given event. Each nova is represented by an excitation function $\rho(M)$, where $M$ is the nova mass. An observed pion in $\pi^{+} \mathrm{p}\left(\pi^{-} \pi^{-}\right)$or $\mathrm{pp}\left(\pi^{-} \pi^{-}\right)$is a decay product of one of the nova systems, but in $\pi^{-} \mathrm{p}\left(\pi^{-} \pi^{-}\right)$it may also be a through-going quasielastically scattered $\pi^{-}$(Fig. 2(b)).

2. Single particle transverse momentum distributions are observed to be damped sharply, following a gaussian like dependence of the form $\exp \left(-\lambda \mathrm{p}_{\mathrm{T}}{ }^{2}\right)$, where $\lambda \approx 3$ for large $\mathrm{p}_{\mathrm{T}}{ }^{2}$, and $\lambda \approx 10$ for small $\mathrm{p}_{\mathrm{T}}^{2}(\leq 0.2)$. Although often neglected, the distribution in $\mathrm{p}_{\mathrm{T}}{ }^{2}$ has non-trivial implications for distributions in $\mathrm{y}$. Indeed, at current accelerator energies, the mean $\left\langle\mathrm{p}_{\mathrm{T}}\right\rangle$ is not small compared to the 
average energy available per secondary. Thus, the fact that a fair fraction of the available energy goes into transverse motion reflects itself in narrow distributions in $\mathrm{x}$ or $\mathrm{y}$. We observe that over the energy range $10-30 \mathrm{GeV} / \mathrm{c},\left\langle\mathrm{p}_{\mathrm{T}}{ }^{2}\right\rangle \simeq\left\langle\mathrm{p}_{\mathrm{L}}{ }^{2}\right\rangle$ in the nova rest frame; this provides justification for our assumption that when clusters, produced with small inelasticity, decay by pion emission, the average decay distribution is approximately isotropic. (29). Another consequence is our assertion that the average number of decay pions $n(\mathbb{M})$ is linearly proportional ${ }^{(30)}$ to the nova mass $M$.

3. A third important feature of single pion inclusive spectra is the observed rapid approach to scaling ${ }^{(28)}$ (energy independence) for intermediate values of $|x|(|x| \approx 0.2$ to 0.6$)$. These energetic secondary pions yet short of being leading particles, tend to result from decay of fairly low-mass novas. High-mass clusters, which can be excited with increasing energy, populate small values of $|\mathrm{x}|$. Rapid scaling is thus achieved if our low M excitation spectrum $\rho(M)$ is energy independent and peaks at relatively small M. Energy independence at small $M$ is supported by the observed ${ }^{(26)}$ near constancy of $\sigma_{4}$ over the energy range 10 to $500 \mathrm{GeV} / \mathrm{c} ; \sigma_{4}$ is the four prong exclusive cross section.

These three crucial features of machine energy collisions are systematized in the Nova model, where either the beam or target particle is quasi-elastically excited into a superposition of resonance 
states which subsequently decay by sequential emmision, with an isotropic decay distribution in the nova rest frame. The mean $\mathrm{p}_{\mathrm{T}}$ of the pion distribution is related to the multiplicity of pions obtained from a nova mass $M$; this in turn fixes the normalization of $d \sigma / d y$. Although a few additional specific technical assumptions are made in setting up the Nova-model ${ }^{(21)}$ calculation," the general shapes of predicted distributions do not depend sensitively on these as sumptions. The crucial assumptions are those stressed above. Insofar as energy dependence is concerned, the way in which we formulate the model leads to strong predictions for $\langle n(n-1)\rangle$ in the NAL and ISR energy ranges. Energy variation is discussed in Section III, but seems immaterial for discussion of $d^{2} \sigma / d y_{1} d y_{2}$ in the energy range 10 to 30 GeV/c. Although perhaps obvious, we stress that the model is not built upon what would be called fundamental theoretical postulates. Rather, we abstract simple observed features from single particle inclusive spectra and then imbed them in a framework which is convenient for further phenomenological analyses. Detailed calculations of shapes of single particle distributions are correct. Interesting tests are possible at the two particle inclusive level, where we can study whether additional dynamics is present, beyond that abstracted from the single particle spectra. Similarly our attitude toward single versus double excitation remains phenomenologically based ${ }^{(21)}$ and no. matter of principles.

* As must be true in all approaches even weighted phase space. 


\section{B. Parametrization}

Relevant diagrams are sketched in Fig. 2. A nova is characterized by three quantities: its excitation spectrum $\rho(\mathbb{M})$, the mean number $n(M)$ of decay pions, and the average decay distribution $\mathrm{dD} / \mathrm{d}^{3} \overrightarrow{\mathrm{p}}$ in the Nova rest frame. In addition, when consider ing leading pion effects, we must specify the momentum transfer $(t)$ or transverse momentum $\left(\mathrm{p}_{\mathrm{T}}\right)$ distribution of the leading particle. The $t$ or $\mathrm{p}_{\mathrm{T}}$ distribution of the whole cluster is irrelevant when we examine pions which are decay products of a cluster. Because of the small value of the pion mass and small $Q$ value in the decay, ${ }^{(21)}$ the convolution of $\mathrm{p}_{\mathrm{T}}$ distributions for production and decay is controlled entirely by the decay distributions $(31,32)$ We discuss leading particle effects in Section II, E. Here we concentrate on parameterization of single nova excitation graphs Fig. 2(a) and (b).

For reasons given above, the average pion decay distribution in the nova rest frame is chosen to have the symmetrical form

$$
\frac{d D}{d^{3} p}=\frac{a}{k^{2} \omega} \exp \left(-\frac{p^{2}}{k^{2}}\right)
$$

Here $\omega=\left(p^{2}+\mu^{2}\right)^{1 / 2}$ is the energy of the secondary $\pi$ in the nova rest frame; $p^{2}=p_{T}{ }^{2}+p_{L}{ }^{2}$; and $\mu$ is the pion mass. Parameter $K$ is chosen to reproduce the correct average single particle $\mathrm{p}_{T}{ }^{2}$ 
distribution. ${ }^{(3,4)}$ We determine a value $\mathrm{K}=0.45 \mathrm{GeV} / \mathrm{c}$, which corresponds to a typical Q value of $330 \mathrm{MeV} / \mathrm{C}$ at each step in the sequential decay of the nova. The distribution is normalized to unity by a.

It will be noted that in Eq. (2.1) the same parameter $K$ controls both longitudinal and transverse momentum spectra, a result supported by our comparisons with data. The form of the decay distribution specifies a fixed average energy release per decay, so that the total number $n(M)$ of decay pions is linearly proportional to the excitation mass ${ }^{(30)}\left(\mathrm{M}-\mathrm{M}_{\mathrm{O}}\right)$. The proportionality factor is also related to the value of $K$, with reasonable values determined to lie in the range 2.0 to $2.5 \mathrm{GeV}^{-1}$. After adjusting results to reproduce the correct mean multiplicity ${ }^{(12)}$ in $\mathrm{pp}\left(\pi^{-}\right)$at $21 \mathrm{GeV} / \mathrm{c}$, we find

$$
h(M)=2.1\left(M-M_{0}\right)
$$

This is understood as an implicit average over all decay chains; $M_{0}$ is the ground state mass of the nova. Although the factor 2.1 was determined for proton novas, Eq. (2.2) is also used for our pion and kaon nova calculations. Looking for key features, we minimize the number of parameters.

In addition to Eq. (2.1), other forms for $\mathrm{dD} / \mathrm{d}^{3} \overrightarrow{\mathrm{p}}$ are possible. We also tried the expression 


$$
\frac{d D}{d^{3} p}=\frac{a^{\prime}}{k^{\prime 2} \mu} \exp \left(-\frac{p^{2}}{k^{\prime 2}}\right)
$$

This differs from Eq. (2.1) in that the factor $\omega^{-1}$ is absent on the right hand side. Here $K^{\prime}=0.33$ is different from $K$, for obvious reasons, but average quantities remain the same. Numerical calculations were performed with both Eqs. (2.1) and (2.3), and agreement was found, indicative of the fact that results in rapidity are not overly sensitive to the exact form for $d D / d \vec{p}$. All specific results reported here were done with Eq. (2.1). Neither Eq. (2.1) nor (2.3) provides a perfect representation of $d \sigma / d p_{T}{ }^{2}$. To achieve better agreement, we should perhaps use a sum of gaussians on the right hand side ${ }^{(33)}$ of Eqs. (2.1) and (2.3). Results in rapidity are insensitive to such precision; it is important but sufficient to reproduce the average behavior of $\mathrm{d} \sigma / \mathrm{dp}_{\mathrm{T}}{ }^{2}$.

Rewriting Eq. (2.1) in terms of rapidity, we obtain

$$
\frac{d D}{d y_{0} d p_{T}^{2}}=\frac{\pi a}{k^{2}} \exp \left(-\frac{p_{T}^{2}}{k^{2}}\right) \exp \left(-\left(\frac{m_{T} \sinh y_{0}}{k}\right)^{2}\right)
$$

where $y_{0}$ is the rapidity in the nova rest frame and $\mathrm{m}_{T}{ }^{2}=\mathrm{p}_{\mathrm{T}}{ }^{2}+\mu^{2}$. (Here we use the relations $\mathrm{p}_{\mathrm{L}}=\mathrm{m}_{\mathrm{T}} \sinh \mathrm{y}_{\mathrm{O}}$ and $\omega=\mathrm{m}_{\mathrm{T}} \cosh \mathrm{y}_{0^{\circ}}$ ) For reasons given above, values of $\mathrm{p}_{\mathrm{T}}$ are essentially identical in the center-of-mass and nova rest frames. To transform 
rapidities, we use their convenient properties under Lorentz transformations. We write

$$
y=y_{0} \pm Y(s, M)
$$

Here $\mathrm{y}$ is the center-of-mass rapidity. The $(+)$ sign is taken for beamlike (e.g. meson) novas and the (-) sign for target novas. Nova rapidity $\mathrm{Y}$ is calculated from

$$
\sinh Y=\frac{Q_{N}}{M}
$$

where $Q_{N}$ is the center-of-mass momentum of the nova.

$$
Q_{N}=\left(S^{2}-2 s\left(M^{2}+M_{P}^{2}\right)+\left(M^{2}-M_{P}^{2}\right)^{2}\right)^{1 / 2} / 2 \sqrt{S}
$$

Here $M_{p}$ is the mass of the "spectator" initial particle.

After integrating Eq. (2.4) over $\mathrm{p}_{\mathrm{T}}$, we obtain

$$
\frac{d D}{d y_{0}} \simeq \frac{a}{\cosh ^{2} y_{0}} \exp \left(-\left(\frac{\mu \sinh y_{0}}{k}\right)^{2}\right)
$$

The form is approximate because in practice the integral must be cut off at finite $\mathrm{p}_{\mathrm{T}}$, in keeping with energy and momentum conservation. We define $A(M, y)$ to be the normalized nova decay distribution in rapidity in the center-of-mass. 


$$
A(M, y)=\frac{d D}{d y_{0}}(y \mp Y)
$$

We turn next to the parameterization of our third quantity, the nova excitation spectrum $p(M)$. This quantity is the differential cross section $d \sigma / d M$, integrated over momentum transfer, for production of a nova of mass $M$. Choice of a functional form for $\rho(\mathrm{M})$ is necessarily somewhat ad hoc. (21) However, because of the averaging (integration) over $M$ required in calculating various distributions, the distribution of secondary pions coming from nova decay is insensitive to details of $\rho(M)$. Our form for $\rho(M)$ rises from threshold $\left(\mathrm{M}=\mathrm{M}_{\mathrm{O}}\right)$, peaks at $\approx 1 \mathrm{GeV}$ above threshold, and falls in proportion to $\left(M-M_{0}\right)^{-2}$ for large $M$. As long as this general rise and fall of $\rho(M)$ is maintained, distributions are not modified greatly.

The asymptotic $\mathrm{M}^{-2}$ behavior is suggested by Regge theory. (34) Given our determination that $n(M) \propto M$, the $M^{-2}$ behavior is also required in order that the mean pion multiplicity grow in proportion to $\log \mathrm{s}$. This logarithmic property is already enough to guarantee a reasonable phenomenological description of single-particle spectra near $\mathrm{x}=0 .{ }^{(25)}$ However, two-particle distributions are sensitive to high mass excitations; ther efore, our estimates of high M behavior are not guaranteed to account correctly for normalization 
of $\mathrm{d}^{2} \sigma / d y_{1} \mathrm{dy}_{2}$.

The form for $p(M)$ is different in general for baryon and meson Novas. The specific form we choose is $(21)$

$$
\rho_{K}(M)=\left(M_{-} M_{K}\right)^{-2} \exp \left(-\beta_{\alpha} /\left(M_{-} M_{\alpha}\right)\right)
$$

The values $\beta_{p}=2 \mathrm{GeV}$ for proton excitations and $\beta_{\pi}=2.4 \mathrm{GeV} / \mathrm{c}$ for pion excitations are found to give good agreement with both singleand two-particle inclusive spectra in $\mathrm{pp}\left(\pi^{-}, \pi^{-}\right)$and $\pi^{ \pm} \mathrm{p}\left(\pi^{-}, \pi^{-}\right)$. We normalize our calculation to the observed total inelastic cross section at a given energy. This introduces normalization constants $\mathrm{C}_{\alpha}$. In addition, to reproduce the fact that secondaries tend to follow the incident meson (an observed asymmetry of single-particle distributions), we set $C_{\pi} / C_{p}=2$, as in Ref. 21 and 22 .

If only single nova excitations contribute to $\pi p(\pi)(e . g$. Fig. 2 (a) and (b) only), then the inelastic cross section is given by

$$
\sigma_{\text {ind }}^{(1)}=C_{0}\left(\int_{m_{p}}^{\sqrt{x}-m_{p}} \rho_{p}(M) d M+2 \int_{m_{\pi}}^{\sqrt{s}-m_{p}} \rho_{\pi}(M) d M\right)
$$

and

$$
\sigma_{\text {ind }}^{(1)}\langle n\rangle^{(1)}=C_{0}\left(\int_{m_{p}}^{\sqrt{s} \cdot m_{n}} n_{p}(n) P_{p}(n) d n+2 \int_{n_{m}}^{\sqrt{s_{m} m_{p}}} n_{\pi}(n) \rho_{\pi}(n) d \eta\right)
$$

Note that $\langle n\rangle$, the average number of pions, is independent of $\mathrm{C}_{0} \cdot$ As already stressed, ${ }^{(21)}$ our keeping the same expression all the way to phase space limits in purely phenomenological. It enforces the correct multiplicity but should not be given a dynamical significance. Our crude spectrum ignores low energy resonances $\left(\rho^{+}, \Delta^{+}\right)$. They do not contribute to the $\pi$ yield but we lump into single excitation cases in which the $\pi(p)$ recoils as a $\leq$ ) while exciting the other particle. 
The quantity $n(M)$ gives the average number of pions which decay from a nova of mass $M$. These pions must be apportioned among the three possible charge states, a procedure detailed in Section III. Here we denote by $\mathrm{n}_{\alpha}^{-}(\mathrm{M})$ the average number of negative pions expected from the decay of an $\alpha$ nova of mass $M$. We introduce $\left.<\mathrm{n}_{\alpha}^{-}\left(\mathrm{n}_{\alpha}^{-}-1\right)\right\rangle_{M}$ which is twice the average number of pairs of $\pi^{-}$ expected from decay of an $\alpha$ nova of mass $M$.

The contents of this subsection are summarized in the following expressions for the single nova contributions to $d \sigma / d y$ and $d^{2} \sigma / d y_{1} d y_{2}$ for $\pi^{-}$production in $M_{1} M_{2}\left(\pi^{-}, \pi^{-}\right)$.

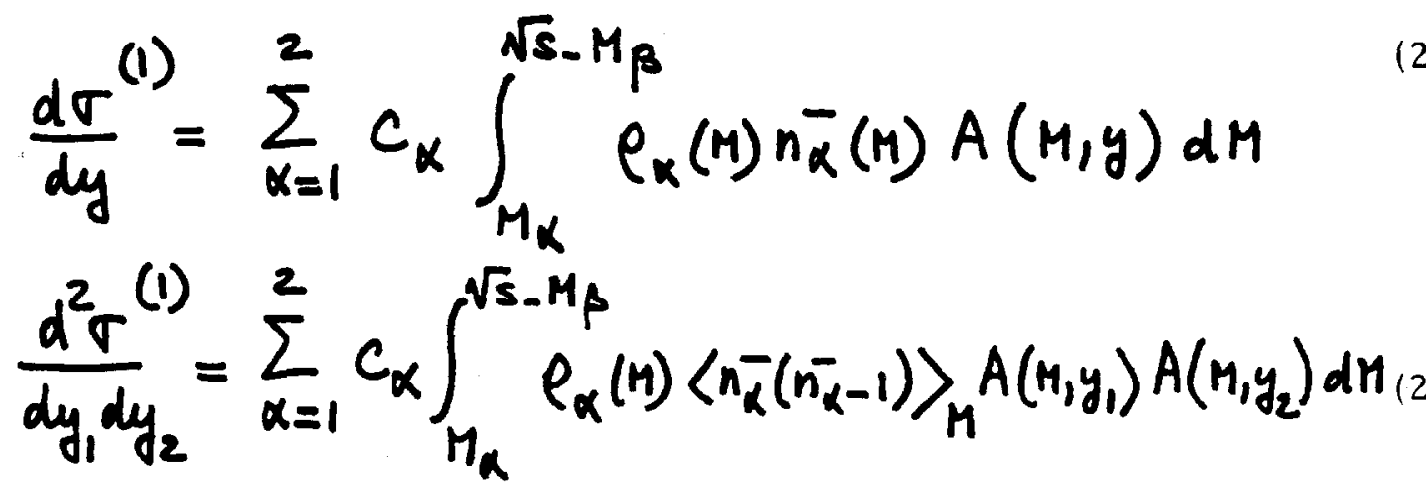

These equations were used to obtain distributions presented in Ref. 22 for $\pi^{+} \mathrm{p}\left(\pi^{-}, \pi^{-}\right)$. As remarked in that paper, the fact that both $\pi^{-}$are always associated with the same nova results in a marked tendency for $d^{2} \sigma / d y_{1} d y_{2}$ to peak at positive values of $y_{2}$ when $y_{1}$ is positive. To the extent that this strong feature is not observed directly in data for large values of $\left|\mathrm{y}_{1}\right|$ it is obvious that, the two observed $\pi^{-}$sometimes originate from two different clusters. In a configuration such 
as that shown in Fig. 2(c), the two novas may decay with pions being emitted in a back-to-back fashion. In the next subsection, we discuss our parameterizations of Fig. 2 (c).

C. Double nova Production.

If we insist that one $\pi^{-}$is produced with large $|\mathrm{y}|$, kinematics suggest that it originates from a light fast nova. Because the probability is small for a nova with small $M$ to give two $\pi^{-}$, it is likely that if a second $\pi^{-}$is observed, it will have come from a different cluster, moving in the opposite direction from the first. Two particle distributions $d^{2} \sigma / d y_{1} d y_{2}$ with one $y$ large are sensitive to this type of double nova excitation (as previously remarked. (22)) It remains however, that single nova excitation (one nova per event) is important.

The width (or dispersion) of d $\sigma / d y$ is determined by low mass novas, which can seldom produce two pions of the same charge. Heavier novas, which can give two $\pi^{-}$, are restricted by kinematics to relatively small values of $y$. Thus, decay pions from heavy novas also occupy the small y region. To the extent that single nova effects dominate, $d^{2} \sigma / d y_{1} d y_{2}$ will be more narrow than $d \sigma / d y$. This is supported by the data in Fig. 3, for small values of $\mathrm{y}_{1}$. However, viewed as a function of $\mathrm{y}_{2}, \mathrm{~d}^{2} \sigma / \mathrm{dy}_{1} \mathrm{dy} \mathrm{y}_{2}$, at large $\mathrm{y}_{1}$ is as broad as $d \sigma / d y$, as expected if two cluster contributions are now significant. Our physical picture then is one in which dominant single nova effects 
supply the bulk of the distributions, particularly at small y. Their contribution is supplemented by double-nova effects, whose role is particularly important in $d^{2} \sigma / d y_{1} d y_{2}$ when either or both $y$ are large.

When two clusters are formed in a given event, it is not obvious that we should describe their excitation and decay in the same way as we handle the single nova case. However, to the extent that the masses of the clusters are small, their pion yields should be similar to those of novas formed in a single excitation configuration. We therefore adopt the same $\rho(M), n(M)$, and so forth.

For the double excitation contribution to $\sigma_{\text {inel }}$, we write

$$
\sigma_{\text {incl }}^{(2)}=\int e_{1}\left(H_{1}\right) e_{2}\left(H_{2}\right) R\left(H_{1}, H_{2}\right) d H_{1} d M_{2}
$$

Here $M_{1}$ and $M_{2}$ are masses of the two novas. Function $R\left(M_{1}, M_{2}\right)$ scales the double excitation cross section as compared to the single excitation contribution, Eq. (2.11). We find it adequate to take a constant value for $R$, followed by a sharp cutoff $(R=0)$ if momentum transfers $t\left(M_{1}, M_{2} ; s\right)$ are larger than $1(\mathrm{GeV} / \mathrm{c})^{2}$.

For the double excitation contribution to $\langle n\rangle$, we derive

$$
\sigma_{\text {inel }}^{(2)}\langle n\rangle^{(2)}=\int e_{1}\left(M_{1}\right) e_{2}\left(M_{2}\right) R\left(H_{1}, M_{2}\right)\left(n_{1}\left(M_{1}\right)+n_{2}\left(M_{2}\right)\right) d M_{1} d M_{2}
$$


$-24-$

For $\pi^{+} \mathrm{p}\left(\pi^{-}\right), \mathrm{pp}\left(\pi^{-}\right), \mathrm{K}^{+} \mathrm{p}\left(\pi^{-}\right)$, therefore,

$$
\langle n-\rangle=\frac{\langle n\rangle^{(1)} \sigma_{\text {ind }}{ }^{(1)}+\langle n\rangle^{(2)} \sigma_{\text {inge }}{ }^{(2)}}{\sigma_{\text {ind }}}
$$

where $\sigma_{\text {inel }}=\sigma_{\text {inel }}^{(1)}+\sigma_{\text {inel }}^{(2)}$. The corresponding double excitation contribution to $d \sigma / d y$ is

$$
\begin{aligned}
\frac{d \sigma^{(2)}}{d y}= & \int e_{1}\left(M_{1}\right) e_{2}\left(m_{2}\right) R\left(m_{1}, M_{2}\right) d m_{1} d M_{2} x \\
& \times\left(n_{1}\left(M_{1}\right) A_{1}\left(M_{1}, y\right)+n_{2}\left(m_{2}\right) A_{2}\left(M_{2}, y\right)\right)
\end{aligned}
$$

The decay functions $A_{i}(M, y)$ are the same as those discussed previously (Eq. (2.9)), except that in the kinematics the spectator mass is now that of the bachelor nova. The two terms appearing in Eq. (2.18) represent the fact that the observed $\pi$ may originate from either of the two clusters formed in the event.

For $d^{2} \sigma / d y_{1} d y_{2}$, we have three terms, which refer to our associating the two observed $\pi$ to two different clusters or to the same one. Specifically,

$$
\begin{aligned}
& \frac{d^{2} \sigma}{d y_{1} d y_{2}}=\int \rho_{1}\left(M_{1}\right) e_{2}\left(M_{2}\right) R\left(M_{1}, H_{2}\right) d M_{1} d M_{2} \times \\
& \times\left\{n_{1}\left(M_{1}\right) n_{2}\left(M_{2}\right)\left(A_{1}\left(M_{1}, y_{1}\right) A_{2}\left(n_{2}, y_{2}\right)+A_{1}\left(M_{1}, y_{2}\right) A_{2}\left(H_{2}, y_{1}\right)\right)\right. \\
& +\left\langle n_{1}\left(n_{1}-1\right)\right\rangle_{H_{1}} A_{1}\left(n_{1}, y_{1}\right) A_{1}\left(n_{1}, y_{2}\right) \\
& \left.+\left\langle n_{2}\left(n_{2}-1\right)\right\rangle_{n_{2}} A_{2}\left(M_{2}, y_{1}\right) A_{2}\left(n_{2}, y_{2}\right)\right\}
\end{aligned}
$$


Furthermore,

$$
\begin{aligned}
& \sigma_{\text {ind }}^{(2)}\langle n(n-1)\rangle^{(2)}=\int e_{1}\left(n_{1}\right) e_{2}\left(n_{2}\right) R\left(n_{1}, n_{2}\right) d n_{1} d n_{2} \times \\
& \times\left\{2 n_{1}\left(m_{1}\right) n_{2}\left(m_{2}\right)+\left\langle n_{1}\left(n_{1}-1\right)\right\rangle_{n_{1}}+\left\langle n_{2}\left(n_{2}-1\right)\right\rangle_{H_{2}}\right\}
\end{aligned}
$$

D. Normalization and Calculated Distributions.

The necessary ratio of $\sigma^{(2)} / \sigma^{(1)}$ [c.f. Eqs. (2.11) and

(2.15)] is determined by fitting shapes of distributions for $\pi^{+} \mathrm{p}\left(\pi^{-}, \pi^{-}\right)$ shown in Fig. 3. The essentially perfect agreement with data is obtained with the value $\sigma^{(2)} / \sigma^{(1)}=3 / 7$. As mentioned above, the double excitation "correction" to dominant single excitation is needed in order to reproduce the width of $d^{2} \sigma / d y_{1} d y_{2}$ when one rapidity is kept large.

The single particle distribution d $\sigma / d y$ for $\pi^{+} \mathrm{p}\left(\pi^{-}\right)$at 18.5

$\mathrm{GeV} / \mathrm{C}$ is shown in Fig. 1. Again, agreement is excellent. Given our determination of $\sigma^{(2)} / \sigma^{(1)}$, we normalize our calculation to the observed inelastic cross section of $21 \mathrm{mb}$. Thus, normalizations of $\mathrm{d} \sigma / \mathrm{dy}$ in Fig. 1 and $\mathrm{d}^{2} \sigma / \mathrm{dy}_{1} \mathrm{dy}_{2}$ in Fig. 3 are predictions of the model.

A similar procedure was pursued ${ }^{(12)}$ for $p p\left(\pi^{-}, \pi^{-}\right)$, a 
cleaner process to analyze inasmuch as only proton novas contribute and the single particle distribution $d \sigma / d y$ is symmetric about $y=0$. Again, a $7 / 3$ ratio of single to double excitation was found to provide excellent agreement with the Michigan State pp data. Results from Ref. 12 are reproduced here as Fig. 4. Also shown in Fig. 4 is the explicit contribution from double excitation to $d^{2} \sigma / d y_{1} d y_{2}$ versus $\mathrm{y}_{2}$, for $\mathrm{y}_{1} \simeq 2.0$. The pp calculation is normalized to the observed $\mathrm{pp}$ inelastic cross section of $30 \mathrm{mb}$ at $21 \mathrm{GeV} / \mathrm{c}$; normalization of $\mathrm{d} \sigma / \mathrm{dy}$ and $\mathrm{d}^{2} \sigma / \mathrm{dy}_{1} \mathrm{dy}_{2}$ are predicted correctly.

Two particle distributions are more narrow than single distributions, and thus give obvious indication of strong positive clustering (correlation) of secondary $\pi^{-}$'s. However, this effect simply reflects the fact that $d^{2} \sigma / d y_{1} d y_{2}$ receives contributions from heavier nova events yielding at least two $\pi^{-}(6$ prongs or more in pp or $\pi^{+} \mathrm{p}$ ), whereas $\mathrm{d} \sigma / \mathrm{dy}$ is populated by a larger sample of events. Over the energy range 10 to $30 \mathrm{GeV} / \mathrm{c}$, the four prong cross section (only one $\pi^{-}$) is particularly large. In terms of the model, production of two $\pi^{-}$requires relatively heavy clusters, thus narrow distributions in $y$ of the decay pions. From a purely experimental point of view, the same conclusion may be reached by comparing the width of $d^{2} \sigma / d y_{1} d y_{2}$ to that of $d \sigma / d y$ limited to events in which at least two $\pi^{-}$are found. Widths are then comparable. 
Another interesting way to examine clustering is to define an associated multiplicity $(12,36)$

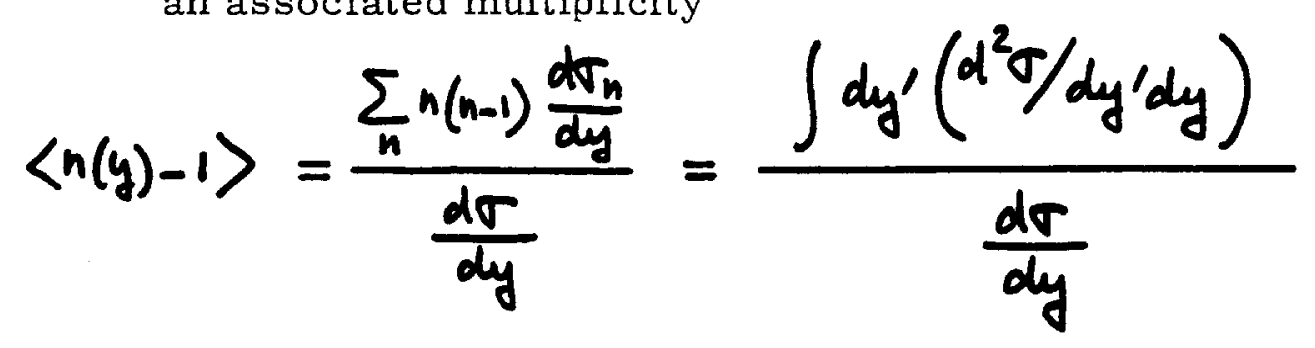

Because the averaged double distribution is narrower in $y$ than the single particle distribution, Eq. (2. 21) should have a maximum near $y=0$. Results of our calculations are compared with Notre Dame data in Fig. 5. Agreement is excellent. This again indicates, through a slightly different test, that our description of the clustering effect is enough to explain observed positive correlations among $\pi^{-}$'s. The observed effect, particularly marked between distributions requiring only one versus those requiring at least two $\pi^{-}$, is due to the relative importance of low mass novas. This effect disappears slowly with increasing $n$, when one compares distributions limited to multiplicity with more than $n$ and $n+1 \pi^{-}$'s respectively.

E. Leading Particle Contribution.

If one of the observed secondaries can be a quasi-elastically scattered particle, as in $\pi^{-} \mathrm{p}\left(\pi^{-}\right)$, an additional corresponding term should be added to the single and double distributions. The leading particle rapidity distribution is obtained in terms of the excitation spectrum of the recoil nova; it reads 


$$
\frac{d \sigma_{L}}{d y}=k \sqrt{s} \int \frac{1}{M_{0}} \frac{d \rho\left(M_{0}\right)}{d t} d p_{T}^{2}
$$

The center of mass momentum is denoted by $\mathrm{k}$; and $\mathrm{M}_{0}$, the recoil nova mass, is readily evaluated in terms of $y$ and $\mathrm{p}_{\mathrm{T}}{ }^{2}$, as

$$
\begin{array}{r}
M_{0}^{2}=s-2 \sqrt{s} m_{T} \sinh y+\mu^{2} \\
\text { with } \quad m_{T}^{2}=P_{T}^{2}+\mu^{2}
\end{array}
$$

For phenomenological reasons we have taken

$$
\frac{d P}{d p_{T}^{2}}=\rho(H) B \exp \left(-B P_{T}^{2}\right)
$$

with $\mathrm{B}=4 \mathrm{GeV}^{-2}$ independent of $\mathrm{M}$.

At fixed $y$, different values of $\mathrm{p}_{\mathrm{T}}{ }^{2}$ correspond to different values of M. Since $\mathrm{y}$ depends very strongly on $\mathrm{p}_{\mathrm{T}}{ }^{2}$ at fixed $\mathrm{M}$, no prominent quasi-elastic peak is obtained (Fig. 1). In order to see the peak clearly, one has to consider separately small $\mathrm{p}_{\mathrm{T}}{ }^{2}$ intervals. (18)

Keeping only single nova excitation and normalizing the calculated distribution to the total inelastic cross section we obtain too strong a leading particle contribution. ${ }^{(21)}$ This is another indication that some double excitation is also present. Granting only $70 \%$ of $\sigma_{\text {inel }}$ to single excitation (as for $\pi^{+} p$ ) we decrease the 
calculated value of the leading pion distribution and obtain a good agreement with the data shown on Fig. 1. Our estimate of the leading particle effect could be an overestimate. (17) The ratio $\sigma^{(2)} / \sigma^{(1)}$ which we used is a compromise which could be modified were the shape and normalization of the leading particle contribution to change with more accurate data. (37) As already emphasized ${ }^{(21)}$ it would be very interesting to examine data on the slow proton recoil distribution which should offer the most reliable determination of the actual strength of single excitation.

To obtain $d \sigma / d y$ for $\pi_{-p}\left(\pi^{-}\right)$we add three contributions, from Eqs. (2.13), (2.18), and (2.22). Our calculation for $\pi-p(\pi-)$, shown in Fig. 1, is normalized to $\sigma_{\text {inel }}(\pi+p) !$ The relative normalization for $\pi^{ \pm} \mathrm{p}$ is a prediction of the model. The discrepancy with data seen for $y<o$ (for $\pi-p$ ) is related to charge exchange effects neglected in our calculation. (21) Such effect which should fall with increasing energy (as opposed to the large difference found at low positive $y$ ) are much larger for $\pi-p$ than for $\pi+p$ processes.

The leading particle also contributes to double distributions. Indeed for $\mathrm{y}_{1}>2$, the observed $\pi^{-}$is in general the quasi-elastically scattered $\pi^{-}$. One finds

$$
\begin{gathered}
\frac{d^{2} \sigma}{d y_{1} d y_{2}}=\int \frac{k \sqrt{s}}{M_{0}} \frac{d e\left(M_{0}\right)}{d t} n_{p}\left(M_{0}\right) A_{p}\left(y_{2}, H_{0}\right) d P_{T}^{2} \\
+(1 \leftrightarrow 2)
\end{gathered}
$$


where $A_{p}\left(y_{2}, M_{0}\right)$ is the pion distribution obtained from the proton nova and $n_{p}\left(M_{0}\right)$ is the average number of $\pi^{-}$from a proton nova of mass $M_{0}$. Depending on the magnitude and sign of $y_{1}$, only one term in (2.25) may be significant in the double distribution.

Upon including contributions from Eqs. (2.14), (2.19), and (2. 25), we obtain results presented in Fig. 6. Although overall agreement with data is less spectacular than for $\pi^{+} p$ and $p p$, results are acceptable for $\mathrm{y}>-0.8$. For $\mathrm{y}<-0.8$, normalization difficulties are particularly evident. However, these can be traced to the problem which we experienced for $\mathrm{y}<0$ in $\mathrm{d} \sigma / \mathrm{dy}$, and which we discussed above. Improving parameters was not attempted.

Our results for $\pi^{ \pm} \mathrm{p}$ and pp processes show that the simple features we abstracted from single particle inclusive data and imbedded in the nova framework are fully sufficient for reproducing more detailed information provided by two particle inclusive reactions. The clustering effect (small inelasticity) inferred from single particle spectra plays an especially significant role at the two particle inclusive level. This is an important result., but, at the same time, we may conclude that beyond confirming features of single particle data, present data 2 on $\mathrm{d}^{2} \sigma / \mathrm{dy}_{1} \mathrm{dy}_{2}$ do not reveal any new specific dynamic phenomenon. 
F. Correlation Function.

It may seem practical to summarize the information which is contained in the double distributions in terms of a correlation function $\mathrm{C}\left(\mathrm{y}_{1}, \mathrm{y}_{2}\right)$. However, its interpretation may not be too transparent at energies where the mean multiplicity of the observed secondaries is 1 or $2 !$ Consequently the shape and normalization of the correlation are quite sensitive to its definition. The conventional definition is

$$
C\left(y_{1}, y_{2}\right)=\frac{1}{\sigma_{\text {ind }}} \frac{d^{2} \sigma}{d y_{1} d y_{2}}-\frac{1}{\sigma_{\text {indel }}^{2}} \frac{d \sigma}{d y_{1}} \frac{d \sigma}{d y_{2}}
$$

At present machine energy however, the second term measured in $\mathrm{pp}\left(\pi^{-}\right)$collision for instance, has a strong contribution from 4 prong events which make no contribution to the first term. As a result one merely sees the difference between two large and weakly correlated terms. This can hardly be called a correlation in a dynamical sense. Calculating $\mathrm{C}\left(\mathrm{y}_{1}, \mathrm{y}_{2}\right)$ from the double and single distributions is a very inefficient way to display the available information.

At the same time $(2.26)$ is too sensitive to the type of reaction or particle species. The first term normalizes to $\langle n(n-1)\rangle$ when the second one normalizes to $\langle n\rangle^{2}$, and it is merely the charge dependence of these normalizations which leads to such different looking correlation functions. $(9-11)$ Indeed from Table I we see that $\mathrm{C}\left(\mathrm{y}_{1}, \mathrm{y}_{2}\right)$ for $\pi^{-} \mathrm{p}\left(\pi^{-}, \pi^{-}\right)$should be about three times that of 
$\pi^{+} \mathrm{p}\left(\pi^{-}, \pi^{-}\right)$even though the basic production mechanisms are the same in both cases. It seems appropriate to at least partly eliminate such effects, despite all the evils attached to still a new definition for the correlation function. We therefore define

$$
C\left(y_{1}, y_{2}\right)=\frac{1}{\sigma_{\text {ind }}\langle n(n-1)\rangle} \frac{d^{2} \sigma}{d y_{1} d y_{2}}-\frac{1}{\sigma_{\text {ind }}^{2}\langle n\rangle^{2}} \frac{d \sigma}{d y_{1}} \frac{d \sigma}{d y_{2}}
$$

We have computed this correlation function and compared it with

the data. (9) As expected from our agreement with data in Figs. 1, 3, and 5 we reproduce very well the observed a priori puzzling curves. The correlation curves are shown on Figure 7. We have used (2. 27), and $C\left(y_{1}, y_{2}\right)$ integrates to zero. This would also be the case were we to use (2. 26) with a Poisson distribution as a good approximation.

III. Pion Multiplicity and Normalization of Correlations.

A. Charge Ratios.

Novas are postulated to decay by sequential emission of pions, a property in keeping with their resonance like character. The average number of decay pions is determined by the nova mass, Eq. (2.2). Quantity $\mathrm{n}^{-}(\mathrm{M})$ in Eqs. (2.13) and (2.18) is the average number of $\pi^{-}$coming from all decay chains of a nova of mass $M$; $\left.<\mathrm{n}^{-}\left(\mathrm{n}^{-}-1\right)\right\rangle_{\mathrm{M}}$ in Eq. (2.14), is twice the average number of pairs of 
$\pi^{-}$. For very large multiplicity, a statistical limit may be approached in which $\left(\pi^{+} \pi^{-}\right)$pairs are twice as frequent as $\left(\pi^{\circ} \pi^{\circ}\right)$ pairs. Extimates of the relative number of $\pi^{+}, \pi^{-}$, and $\pi^{\circ}$ at low multiplicity depend crucially on the total charge of the nova. Values can be bounded by isospin arguments, but are nonetheless somewhat arbitrary. Results given below are representative of typical decay chains. The appropriateness of our procedure is verified by the fact that we predict approximately the correct normalization of $d \sigma / d y$ for $\pi^{-} p\left(\pi^{-}\right)$at positive $y$ in terms of that for $\pi^{+} \mathrm{p}\left(\pi^{-}\right)$(c.f. Fig. 1).

For large multiplicity, we consider a binomial distribution with $2 / 3$ probability for a $\left(\pi^{+} \pi^{-}\right)$pair and $1 / 3$ probability for a $\pi^{0} \pi^{0}$ ) pair. A straightforward calculation gives

$$
n^{-}(M)=\frac{n(M)}{3} \equiv \frac{N}{3}
$$

for the average number of $\pi^{-}$. Here $N \equiv n(M)$ is the average number of pions of all charges (taken as $n(M)=2.1\left(M-M_{0}\right.$ ) in Section II). The average number of pairs of $\pi^{-}$is

$$
\left\langle n^{-}\left(n^{-}-1\right)\right\rangle_{M}=\frac{N(N-2)}{9}
$$

For low multiplicity (small $M$ ), charge effects are relevant. Proton and $\pi^{+}$novas produce fewer $\pi^{-}$than Eq. (3.1) and $\pi^{-}$novas give correspondingly more. For positively charged novas, reasonable interpolations are 


$$
n^{-}(n)=\frac{N-1}{3}
$$

and

$$
\left\langle n^{-}\left(n^{-}-1\right)\right\rangle_{M}=\frac{(N-1)(N-3)}{9}
$$

Comparisons with some explicit decay chains show that these approximations are quite decent. In calculations of $\pi^{+} p\left(\pi^{-} \pi^{-}\right)$and $p p\left(\pi^{-}, \pi^{-}\right)$ reported in Section II, Eqs. (3.3) and (3.4) were employed.

For negatively charged novas, one extra $\pi^{-}$should be added to the right hand side of Eq. (3.3), a result obtained most simply from a consideration of charge conservation. Thus, for the number of $\pi^{-}$from $\pi^{-}$novas, we use

$$
n^{-}(n)=\frac{N+2}{3}
$$

and

$$
\left\langle n^{-}\left(n^{-}-1\right)\right\rangle_{n}=\frac{(N+3)(N-1)}{9}
$$

Our estimates are crude, but from an examination of explicit decay chains, we estimate a $20 \%$ reliability in $\langle n(n-1)\rangle_{M}$ for $\mathrm{N} \leq 6$, adequate for our purposes. Inasmuch as all quantities of interest are computed averages over $n(M) \rho(M)$ or $\langle n(n-1)\rangle{ }_{M} \rho(M)$, a more refined approach is not justified until $\rho(M)$ is given a more 
exact form.

As described in Section II, Eqs. (3.3) to (3.6) lead to good agreement with shapes and normalization of $d \sigma / d y$ and $d^{2} \sigma / d y_{1} d y_{2}$ at fixed s. We now turn to the energy dependence of $\langle n\rangle$ and $<n(n-1)>$, quantities which normalize $d \sigma / d y$ and $d^{2} \sigma / d y_{1} d y_{2}$ (c.f. Eqs. (1.1) and (1.2), respectively. The quantity $\langle n(n-1)\rangle$ is more model dependent than the shapes of spectra.

B. Energy Dependence.

Our calculated values of $\langle n\rangle$ and $\langle n(n-1)\rangle$ are compared with $18.5 \mathrm{GeV} / \mathrm{c} \pi^{ \pm} \mathrm{p}$ data in Table I. Calculated values of $\langle\mathrm{n}\rangle$ and $f_{2}=\left[\langle n(n-1)\rangle-\langle n\rangle^{2}\right]$ are compared with pp data in Figs. 8(a) and (b). Agreement with $\pi^{ \pm} p$ and $p p$ data in the 10-30 GeV/c energy range is quite respectable.

As remarked in Section II, our model is constructed to give a mean multiplicity $\left\langle\mathrm{n}^{-}>\right.$which grows logarithmically. Indeed, at asymptotic energies, we predict $\left\langle\mathrm{n}^{-}\right\rangle \sim 0.7 \log \mathrm{s}$. For $\langle\mathrm{n}(\mathrm{n}-1)\rangle$, the nova model, in common with all the diffractive fragmentation models, $(2,29)$ predicts an asymptotic increase with energy of the form $\langle n(n-1)\rangle \propto \sqrt{5}$. By contrast, in multiperipheral $(38,39)$ and other weak correlation models $\langle\mathrm{n}(\mathrm{n}-1)\rangle \propto \log \mathrm{s}$, or $\log \mathrm{s}^{2}(8)$

Referring to our discussion of the nova model in Section II. A, we observed that the prediction $\langle n(n-1)\rangle \propto \sqrt{\mathrm{s}}$ is actually not demanded by our phenomenological input. Beginning with the fact that symmetry * In our model <n(n-1) $\rangle_{M} \rho(M)$ goes to a constant at large $M$; thus $\left\langle\mathrm{n}(\mathrm{n}-1)>\alpha \int \mathrm{d} M=\sqrt{\mathrm{s}}\right.$. 
in the nova decay frame suggests $n(M) \propto M$, we chose $\rho(M) \propto M^{-2}$ to guarantee $\left\langle\mathrm{n}>\propto \log \mathrm{s}\right.$ (and an asympotically energy independent $\sigma_{\text {inel }}$ ). However, symmetry is almost forced on us by small inelasticity and the universal $\mathrm{p}_{\mathrm{T}}$ dependence only for reasonably small $\mathrm{p}_{1 \mathrm{ab}}(<100 \mathrm{GeV} / \mathrm{c})$, i. e. for $M$ not infinitely large. We have no way of knowing whether $n(M) \propto \log M\left(\right.$ and $\left.\rho(M) \sim M^{-1}(\log M)^{-1}\right)$ would be more appropriate at very large $M .{ }^{(40)}$ This form would be suggested if the "decay" takes on a one-dimensional character ${ }^{(20)}$ in the nova rest frame, as might be appropriate at very large $\mathrm{s}$ where $\left.\left\langle\mathrm{p}_{\mathrm{L}}\right\rangle\right\rangle\left\langle\left\langle\mathrm{p}_{\mathrm{T}}\right\rangle\right.$.

For reasons of phenomenological simplicity, we stick to $\rho(M) \propto M^{-2}$, for all $M$ at all values of $s$. Thus, we are able to make predictions not only for asymptotic s dependence of $\langle n\rangle$ and $\langle n(n-1)\rangle$, but we can also give specific values for these quantities in the ISR and NAL energy ranges. Some support for the relevance of fragmentation models at ISR energies comes from the observation that in pp collisions the large $\left(\pi^{+} / \pi^{-}\right)$ratio is consistent with being energy independent at

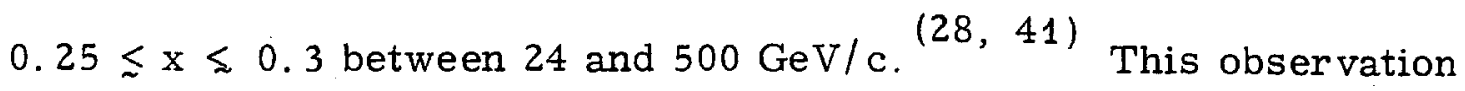
has, however, very little implication for multiplicity growth.

Predicted values for $\langle n\rangle$ and $\langle n(n-1)\rangle$ for $\pi^{ \pm} p$ at $100 \mathrm{GeV} / \mathrm{c}$ are given in Table II. Here $n$ always refers to $\pi^{-}$production. For pp collisions, results given as a function of energy in Figs. 8(a) and (b), are contrasted with available data. (42) Note that our predicted multiplicity distribution, which is narrower than Poisson $\left(f_{2}<0\right)$ in the energy *If this is true $\mathrm{f}_{2}$ would increase logarithmically after an inital sharper rise. 
range $p_{\text {lab }}<30 \mathrm{GeV} / \mathrm{c}$, has become broader than Poisson $\left(\mathrm{f}_{2}>0\right.$ ) by $100 \mathrm{GeV} / \mathrm{c}$. This occurs for both $\mathrm{pp}$ and $\pi^{ \pm}$.

In Fig. 8, our values for $\langle n\rangle$ and $\langle n(n-1)\rangle$ are in reasonable agreement with preliminary Serpukhov data ${ }^{(43)}$ at $69 \mathrm{GeV} / \mathrm{c}$. For $<n>$, we are one to two standard deviations too high in comparison with Echo Lake data ${ }^{(26,44)}$ and too low in comparison with ISR data. However, both experiments are subject to some systematic error. Our most obvious disagreement with present data is seen clearly in Fig. 8(b), where we are a factor of 5 or 10 standard deviations too high in comparison with Echo Lake results ${ }^{(44)}$ on $\mathrm{f}_{2}$ at $200 \mathrm{GeV} / \mathrm{c}$. If these Echo Lake data are correct, they would seem to rule out absolutely the predicted s dependence $\langle n(n-1)\rangle \propto \sqrt{s}$. As remarked above, this would eliminate all pure fragmentation models, ${ }^{(2,29)}$ unless they abandon claim on present machine energy results. Our quantitative predictions for $\langle\mathrm{n}(\mathrm{n}-1)\rangle$, based on specific fragmentation model which reproduces data in the 10-30 GeV/c range very well, underscore the crucial importance of early measurements of $\langle n(n-1)\rangle$ at NAL and ISR. $(22,23)$ More important than statistical accuracy is the obvious need to avoid systematic bias for cross-sections with large prong number, a bias which could significantly raise or lower $\langle n(n-1)\rangle$. IV. Other Correlations and Discussion.

The general ideas and specific procedures described in Sec. II 
and III can be applied easily to other cases to check if the picture of particle production which has been incorporated into the Nova model is generally applicable. We ther efore comment on a number of different correlation experiments.

Our detailed study of two $\pi^{-}$correlations was motivated by the availability of experimental information and supported by the special interest offered by an exotic system. There are no strong final state interactions superimposed on the production process proper. This latter point would also apply to $\mathrm{K}^{+} \mathrm{p}\left(\mathrm{K}^{\circ}, \pi^{-}\right)$reactions. The relatively low yield of $\mathrm{K}^{\circ}$ from proton novas, compared to $\mathrm{K}^{+}$novas, causes the distributions to be shifted to positive kaon rapidities but, otherwise, many features, already found in two $\pi^{-}$correlations, should also be met. We have calculated double rapidity distributions for the reaction $\mathrm{K}^{+} \mathrm{p}\left(\mathrm{K}^{\mathrm{O}}, \pi^{-}\right)$at $16 \mathrm{GeV} / \mathrm{c}$ and present them in Fig. 9. We have used the equations of Section II and III. The number of $\mathrm{K}^{\circ}$ obtained from $\mathrm{K}^{+}$and proton novas respectively are taken to be ${ }^{(46)}$ -

$$
\begin{aligned}
& n_{K+}^{K^{0}}(M)=\frac{1}{2} \\
& n_{P}^{K^{0}}(M)=0.06+0.0 \lg _{p}\left(M-M_{t h}\right)
\end{aligned}
$$

Where $M_{t h}$ is the threshold mass for decay of a proton into a $K^{\circ}$ and 
$\Sigma^{+}$system. The Kaon excitation spectrum is assumed to have the same general shape already used for pion and proton excitations. Just as for pion induced reactions, the ratio $\mathrm{C}_{\mathrm{K}} / \mathrm{C}_{\mathrm{p}}$ is 2 . We present this calculation in order to stimulate a more detailed comparison with possible data. Hypercharge exchange should provide relatively larger correction terms than those met with pion induced reactions (Fig. 1).

Correlations for $\pi^{+} \pi^{-}$or $\overline{\mathrm{K}}^{\circ} \pi^{-}$systems would be interesting. They should show some resonance effects but confirm the main features already found for two pions with the same charge. At present machine energy $\pi^{+} \pi^{-}$double distributions will be normalized at a much larger value. Correlations between final baryon and meson (or two baryons in pp collisions) would also be extremely useful. They would in particular provide further insight on the leading particle effect.

The use of rapidities instead of the Feynman scaling variable $x$ has some obvious kinematical advantages since the peak at small rapidities is compressed into an even sharper peak in an $\left(x_{1}, x_{2}\right)$ distribution. On the other hand, the $x$ variable is better for examining leading particle effects because of the more accurate determination of the nova mass for fixed $x$, expected after averaging over $\mathrm{p}_{T}{ }^{2}$. In any case the strongly peaked shape of the two pion distributions in 
$x_{1}$ and $x_{2}$ may show directly some interesting features. (47) Because of our success with the rapidicy distribution we expect an equally good quantitative representation of the main features when displayed in terms of $x_{1}$ and $x_{2}$. In particular we may draw contour maps in $x_{1}, x_{2}$ for a 3 dimensional presentation of the logarithm of the inclusive double distribution. It is remarkable that at $16-20 \mathrm{GeV}$ we obtain an approximately pyramidal structure at relatively low $\mathrm{x}$ values with roughly planar surfaces in the separate quadrants $\left(x_{1}>0 ; x_{2} \geq 0\right)$.

Our model does not predict dynamical correlations between the transverse momenta of the secondaries (two $\pi^{-}$in section II). The nova decay is statistical and the inclusive distributions approximately factorize in $y$ and $p_{T}{ }^{2}$. As a result $d^{2} \sigma / d p_{T 1}^{2} d p_{T 2}^{2}$ should generally peak at $\mathrm{p}_{\mathrm{T}_{2}}^{2}=0$ for any small value of $\mathrm{p}_{\mathrm{T}_{1}}^{2}$. Nevertheless, if we associate secondaries with large $p_{T}^{2}$ to long jump decays, ${ }^{(33)}$ some correlations could show up. Since long jump decays are relatively infrequent down each decay chain, the $\mathrm{p}_{\mathrm{T}_{2}}^{2}$ distribution at fixed and relatively large $\mathrm{p}_{\mathrm{T}_{1}}^{2}$ should be narrower then when averaged over all observed $\mathrm{p}_{\mathrm{T}_{1}}^{2}$ values. In general however little correlation is expected besides that imposed by energy conservation.

Correlations in $\phi$ have been studied in several cases already $\mathrm{K}^{+} \mathrm{p}\left(\mathrm{K}^{\circ}, \pi^{-}\right)^{(10)}, \mathrm{K}^{-} \mathrm{p}\left(\overline{\mathrm{K}}^{\mathrm{O}}, \pi^{-}\right)^{(48)}$ and $\mathrm{pp}\left(\pi^{-}, \pi^{-}\right) !^{(12,49)}$ In all reactions 
little correlation in $\phi$ has been observed beyond that expected from phase space considerations. Reported results are in agreement with what expected from the Nova model. (49) This may test our sequential decay picture but the observed features are presumably even more general.

As $\mathrm{p}_{\mathrm{T}}{ }^{2}$ is. increased, the distribution should fall more rapidly in $\mathrm{y}$, at least in the range of validity of Eq. $1\left(\mathrm{p}_{\mathrm{T}}^{2}<0.3\right)$. Thus, the rapidity correlations restricted to larger $\mathrm{p}_{\mathrm{T}}^{2}\left(\mathrm{p}_{\mathrm{T}}^{2}>0.1\right)$ should be much stronger (fall off more sharply with $y$ ) than those for smaller $\mathrm{p}_{\mathrm{T}}$. This expectation is almost obvious from the single particle rapidity distribution as a function of $p_{T}^{2}$. This effect is observed experimentally. (9)

In conclusion, it appears that the small inelasticity, rapid scaling and universal $\mathrm{p}_{\mathrm{T}}^{2}$ distributions observed at present accelerator energies are adequate for understanding the key features of correlation data. No further dynamical feature seems to be of any relevance within present accuracy. The Nova model, which embodies this features and provides a practical calculation scheme, appears successful. As already stressed, measurements of $\langle n\rangle$, and in particular of $<n(n-1)\rangle$, performed with increasing ener gy would provide extremely useful information. The strong clustering effect, ascertained at present machine energy, may continue to hold at NAL energy or gradually disappear into a multi-clustering mechanism with weaker correlations which could become dominant at much higher energies. 


\section{ACKNOWLEDGEMENT}

This work would not have been possible without the interest and close cooperation of experimentalists at Notre Dame and Michigan State Universities. We are especially grateful to Jim Powers and Bill Shephard of Notre Dame and to Gerry Smith and Ben Oh of Michigan State University. 


\section{REFERENCES}

${ }^{1}$ R. P. Feynman, Phys. Rev. Letters, 23, 1415 (1969).

${ }^{2}$ T. Benecke, T. T. Chou, C. N. Yang and E. Yen, Phys. Rev. 188, 2159 (1969).

${ }^{3}$ For a review of the experimental situation, see for example M. Deutschmann, Rapporteur's talk, Amsterdam Conference (1971). T. Ludlam, Invited talk at the Cambridge meeting of the American Physical Society (1971), Yale preprint (1972).

${ }^{4}$ E. L. Berger, Argonne Report ANL/HEP 7134, published in Proceedings of the Helsinki Conference, (1971) (ed. by B. Byckling et al. ); E. L. Berger, Argonne Report ANL/HEP 7148; D. Horn, Physics Report, to be published; W. Frazer et al. Rev. Mod. Phys., to be published.

${ }^{5}$ Instead of rapidity $\mathrm{y}$, the Feynman scaling variable $\mathrm{x}$ is often used: $x=2 p_{L} / \sqrt{s}$, where $p_{L}$ and $\sqrt{s}$ are the center of mass Iongitudinal momentum of the secondary and total energy respectively. The rapidity is defined as $\left.y=\ln \left(p_{L}+\left(p_{L}^{2}+m_{T}^{2}\right)^{1 / 2}\right) / m_{T}\right)$ where $m_{T}$ is the transverse mass $m_{T}^{2}=p_{L}^{2}+p_{T}^{2}$.

${ }^{6}$ A. Mueller, Phys. Rev. D2, 2963 (1970).

${ }^{7}$ H. M. Chan, C. S. Hsue, C. Quigg and J. MI. Wang, Phys. Rev. Letters 26, 672 (1971); J. Ellis, J. Finkelstein, P. Frampton and M. Jacob, Phys. Letters 35B, 227 (1971); S.-H.-H. Tye and 
G. Veneziano, Physics Letters 38B, 30 (1972). Unfortunately such rules do not provide an energy scale from which the rate of approach to scaling can be obtained.

${ }^{8}$ E. Predazzi and G. Veneziano, Lettere al Nuovo Cimento 2, 749 (1971);

S. H. H. Tye and G. Veneziano, Phys. Letters 35B, 355 (1971), M. Le Bellac, Lettere al Nuovo Cimento 2, 437 (1971); J. Ellis, J. Finkelstein and R. Peccei, SLAC-PUB-1020 (1972).

${ }^{9}$ W. D. Shephard, J. Powers, N. Biswas, N. Cason, V. Kenney and D. Thomas, Phys. Rev. Letters 28, 703 (1972); W. D. Shephard, Davis Conference (1972). We are much indebted to Jim Powers and Bill Shephard for many discussions about their data.

${ }^{10}$ S. Stone, T. Ferbel, P. Slattery and B. Werner, Rochester Report UR-875-439 (1971).

${ }^{11}$ W. Ko, Phys. Rev. Letters, 28. 935 (1972).

${ }^{12}$ E. L. Berger, B. Oh and G. A. Smith, Argonne preprint ANL/HEP 7209, submitted to Phys. Rev. Letters.

${ }^{13}$ H. D. I. Abarbanel, Phys. Rev. D多, 2227 (1971).

${ }^{14}$ R. Arnold and S. Fenster, Argonne Report ANL/ HEP 7114; C. L. Jen et al., Phys. Rev. Letters, 27, 458 (1971).

${ }^{15}$ R. C. Hwa, Oregon preprint (1971). Invited paper, Davis Conference (1972). 
${ }^{16}$ K. Wilson, Cornell preprint CLNS-131 (1970).

${ }^{17}$ The distribution at large rapidity corresponds to quasi-elastically scattered $\pi^{-}$but the data also have some contamination from elastic events. A clean distribution at large momentum would be extremely useful since it would determine accurately the strengths of single excitation as opposed to more complicated processes.

${ }^{18}$ Aachen-Berlin-Bonn-CERN-Cracow-Heidelberg-Warsaw Collaboration. Amsterdam Conference (1971); J. Beaupre et a1. , Physics Letters 37B, $432(1971)$.

${ }^{19}$ E. W. Anderson et al. , Phys. Rev. Letters 16, 855 (1966); 25, 699 (1970); J. V. Allaby et al., Physics Letters 34B, 435 (1971).

${ }^{20}$ E. L. Berger and A. Krzywicki, Phys. Letters 36B, 380 (1971).

${ }^{21}$ M. Jacob and R. Slansky, Phys. Letters 37B, 408 (1971); Phys. Rev. D5, (1972), Yale preprint (1971).

${ }^{22}$ E. L. Berger and M. Jacob "Two Particle Correlations and Inclusive Reactions" ANL/ NAL preprint (1972)

${ }^{23}$ This ratio differentiates strongly correlated fragmentation models where it increases with energy as $\sqrt{s} / \log ^{2} s$ from weakly correlated (multi-peripheral) models where it tends to a constant with increasing energy. This point has been emphasized by G. F. Chew and C. N. Yang. ${ }^{24}$ We use throughout the short hand notation $A B(C)$, for $A+B \rightarrow C+$ Anything, 
and $A B(C, D)$ for $A+B \rightarrow C+D+$ Anything.

${ }^{25}$ Any model giving an asymptotic logarithmic increase of the multiplicity should reproduce reasonably well the small rapidity (large excitation mass) behavior of inclusive distributions. Since correlations put more weight than single distributions on the higher mass region of the excitation spectrum, they provide a much finer probe.

${ }^{26}$ D. E. Lyon et a1., Phys. Rev. Letters 26, 728 (1971); and L. W. Jones et al. Michigan preprint UMHE 71-46 Rev. (1972).

${ }^{27}$ A. Bialas and K. Zalewski, Cracow preprint (1971).

${ }^{28}$ L. G. Ratner et al. Phys. Rev. Letters 27. 68 (1971) and Rochester APS meeting (1971); see also R. S. Panvini et al., Physics Letters 38B, 55 (1972).

${ }^{29}$ The leading role played by the diffractive excitation of clusters with isotropic decay has been stressed by R. Hwa and C. Lam, Phys. Rev. Letters 27, 1098 (1971); R. Hwa, San Francisco APS meeting (1972) and by R. Adair, Phys. Rev. 172, 1370 (1968), Yale preprint (1972).

${ }^{30}$ Such a feature is also present in the statistical bootstrap model. S. Frautschi, Phys, Rev. D多, 2821 (1971); S. Frautschi and Hamer Phys. Rev. D壬, 2125 (1971).

${ }^{31}$ E. Yen and E. L. Berger, Phys. Rev. Letters 24, 695 (1970). ${ }^{32}$ For heavier secondaries such as protons on $\Lambda^{\prime} s$, the transverse motion of the nova is no longer negligible. 
${ }^{33}$ The wider transverse momentum distribution at large $p_{T}^{2}$ can be associated with "long jump" decays, which are ignored in this paper.

${ }^{34}$ C. E. de Tar et al. , Phys. Rev. Letters 26, 675 (1971); H. D. I. Abarbanel, G. F. Chew, M. Goldberger and L. Saunders, Phys. Rev. Letters 26, 937 (1971). For a practical application see P. Chliapnikov et al., Phys. Letters 36B, 235 (1971).

${ }^{35}$ R. S. Panvini (Vanderbilt), Private Communication. We are much indebted to $R$. Panvini for many informative discussions.

${ }^{36}$ H. Nieh and J. M. Wang, Stony Brook preprint (1971).

37 The data of reference 18 have only a very small contamination if at all from elastic events. We thank D. Morrison (CERN) for a discussion of this point.

${ }^{38}$ G. F. Chew in multiperipheral dynamics, Coral Gables Conference (1971) (Gordon and Breach, N. Y.). L. Bertocchi et al., Nuovo Cimento 25, 626 (1962).

${ }^{39}$ A. Mueller, Phys. Rev. D41, 150 (1971).

${ }^{40}$ We are indebted to $G$. Veneziano for a discussion on this point.

${ }^{41}$ A. Bertin et al., Phys. Letters 38B, 260 (1972).

${ }^{42}$ In all calculations reported in this section, we retain a $7 / 3$ ratio for single to double excitation. Except for leading particle effects, distributions are not extremely sensitive to its value which could be varied slightly. 
${ }^{43}$ Soviet-French Collaboration, preprint (1972) - Mirabelle data.

${ }^{44}$ To obtain $\left\langle\mathrm{n}^{-}\right\rangle$from the results of Ref. 26 and 43 , we take $\left.\left\langle n^{-}\right\rangle=0.5\left(<n^{c h}\right\rangle-2\right)$. Values of $f_{2}$ are computed from tables of prong cross sections given in these two papers.

${ }^{45}$ G. Neuhofer et al., Phys. Letters 37B, 438 (1971) and Phys. Letters 38B, 51 (1972). From this $p p(\gamma)$ experiment, $\left\langle\mathrm{n}^{\circ}\right\rangle$, is obtained. We then assume that $\left\langle\mathrm{n}^{-}\right\rangle=\left\langle\mathrm{n}^{\circ}\right\rangle$, which should not be unreasonable for large values of the multiplicity, although $\left\langle\mathrm{n}^{-}\right\rangle=\left\langle\mathrm{n}^{\mathrm{O}}\right\rangle-0.3$ might be better. We are grateful to G. Charlton (Argonne) for his comments on the $\gamma$ data.

${ }^{46}$ M. Jacob, R. Slansky and C. C. Wu, Yale preprint (1972).

${ }^{47}$ We are grateful to C. Quigg (Stony Brook) for comments on this point. Correlations in $\mathrm{x}$ are discussed in $\mathrm{R}$. Slansky Invited Talk APS meeting, Washington (1972).

${ }^{48}$ We are grateful to $\mathrm{T}$. Ludlam (Yale) for many informative discussions. ${ }^{49}$ M. C. Foster, D. Z. Freedman, S. Nussinov, J. Hanlen and R. S. Panvini, Stony Brook preprint (1972). 


\section{FIGURE CAPTIONS}

Figure 1 Single particle inclusive rapidity distribution for $\pi^{ \pm} \mathrm{p}\left(\pi^{-}\right)$at $18.5 \mathrm{GeV} / \mathrm{c}$. Data are from Notre Dame (Ref. 9). Solid curves are Nova model calculations described in the text. Calculations are normalized to the $\pi^{+} p$ inelastic cross section: $21 \mathrm{mb}$. The relative normalization of the two distributions is a prediction of the model. The difference between the two sets of data at large negative rapidity (proton fragments) is due to charge exchange effects not included in our model calculation. We also did not include some rho production obviously effective at large rapidity.

Figure 2 Three contributions to the inelastic cross section. In (a) an incident meson is excited into a meson nova. The incident baryon loses momentum but is not excited. In (b), the incident baryon is excited into a baryon nova but the meson is quasi-elastically scattered. In (c), both meson and baryon are excited. As a first approximation we keep only terms involving no quantum number exchange.

Figure 3 Two $\pi^{-}$inclusive distributions observed in $\pi^{+} \mathrm{p}\left(\pi^{-}, \pi^{-}\right)$at $18.5 \mathrm{GeV} / \mathrm{c}$. Data are from Notre Dame (Ref. 9). $d^{2} \sigma / d y_{1} d y_{2}$ is plotted versus $y_{2}$ for the following intervals of $y_{1}$ : 
On the left
a) $-0.2<y_{1}<0.2$;
b) $-0.6<y_{1}<-0.2$;
c) $-1.0<y_{1}<-0.6$;
d) $-1.4<y_{1}<-1.0$
e) $-1.8<y_{1}<-1.4$
f) $-2.2<y_{1}<-1.8$ and g) $-2.6<y_{1}<2.2$. On the $r$ ight
h) $0.2<y_{1}<0.6$;
i) $0.6<y_{1}<1$; j) $1<y_{1}<1.4$; k) $1.4<y<1.8$;
1) $1: 8<y_{1}<2.2$
m) $2.2<y_{1}<2.6$ and $\left.n\right) 2.6<y_{1}<3$.
Solid curves are obtained from the Nova model with $70 \%$ of $\sigma_{\text {inel }}$ associated with single nova excitation $\mid$ Fig. $2(a)$ and $(b) \mid$; the rest is double excitation (Fig. 2(c)). Only some of the curves have been drawn.

Figure 4 Experimental distributions and Nova model calculations for $\mathrm{pp}\left(\pi^{-},{\pi^{-}}^{-}\right)$at $21 \mathrm{GeV} / \mathrm{c}$. Data are from Ref. 12. Double distributions are plotted versus $y_{2}$ for values of $y_{1}$ in the intervals indicated. Good agreement is obtained with the same $7 / 3 \mathrm{ratio}$, used for $\pi^{+} p\left(\pi^{-}, \pi^{-}\right)$. The dashed curve represents the double excitation contribution which is obtained for $1.6<y_{1}<2.4$ with $\pi^{-}$in a back to back configuration.

Figure 5 Distributions for $\pi^{-p}\left(\pi^{-}, \pi^{-}\right)$at $18.5 \mathrm{GeV} / \mathrm{c}$. Data from Notre Dame (Ref. 9) are plotted for the following interval of $y_{1}$ : On the left
a) $-0.2<y_{1}<0.2$
b) $-0.6<y_{1}<-0.2$;
c) $-1.0<y_{1}<-0.6$;
d) $-1.4<y_{1}<-1: 0$
e) $-1.8<y_{1}<-1.4$
f) $-2.2<y_{1}<-1.8$ and g) $-2.6<y_{1}<-2.2$. On the 
right: h) $0.2<\mathrm{y}_{1}<0.6$; i) $0.6<\mathrm{y}_{1}<1.0$; j) $1.0<\mathrm{y}_{1}<1.4$; k) $1.4<\mathrm{y}_{1}<1.8$;

1) $\left.1.8<y_{1}<2.2 ; m\right) 2.2<y_{1}<2.6$ and $\left.n\right) 2.6<y_{1}<3.0$.

Theoretical curves are drawn for $\mathrm{y}_{1}=0.4,1.2,1.6,2.0,2.4$ and 2.8 on the right for $\mathrm{y}_{1}=0 .,-0.8,-1.2,-1.6,-2.0$ and -2.4 on the left.

Discrepancies can be related to charge exchange effects as already evident on Fig. 1. Normalization as well as shapes are predictions of the model.

Figure 6 Associated multiplicity, as defined in the text, computed for $\pi^{-}$production in $\pi+p$ collisions at $18.5 \mathrm{GeV} / \mathrm{c}$. The curve is a prediction of the model. Data points are from Ref. 9. The agreement thus met contributes to show that the clustering effect, already inferred from the analysis of single particle distributions, is enough to predict the observed correlation effects.

Figure 7 The correlation function $\mathrm{C}\left(\mathrm{y}_{1}, \mathrm{y}_{2}\right)$ as defined in the text (Eq. 2. 27) plotted versus $y_{2}$ for the following values of $y_{1}$ $\mathrm{y}_{1}=-2.0 ;-0.8 ; 0 ; 0.8$ and 2.0 . The strong positive correlation when both rapidities are small results from our associating the two $\pi^{-}$with a heavy nova. 
Figure 8(a) Mean number of $\pi^{-}$for inelastic pp collision as a function of laboratory momentum. Data are from Michigan State (Ref. 12), Serpukhov (Ref. 43), Echo Lake (Ref. 26) and ISR (Ref. 45). For both Serpukhov and Echo Lake, we use $\left\langle n^{-}\right\rangle=0.5\left(\left\langle n^{c h}\right\rangle-2\right)$ and for ISR we take $\left\langle\mathrm{n}^{-}\right\rangle=\left\langle\mathrm{n}^{\circ}\right\rangle$, assuming that all $\gamma$ rays come from $\pi^{\circ}$ decay. The solid curve is a prediction of the Nova model as described in the text. The mean multiplicity is severely determined by the $\mathrm{p}_{\mathrm{T}}^{2}$ and $\mathrm{y}$ distributions.

Figure 8(b) Quantity $f_{2}=\left\langle n^{-}\left(n^{-}-1\right)\right\rangle-\left\langle n^{-}\right\rangle^{2}$ as a function of $p_{1 a b}$. Both numbers refer to inelastic collisions. The solid curve is a prediction of the Nova model. Echo Lake and Serpukhov points are derived from prong cross sections. Were our disagreement with Echo Lake considered serious, it would mean that the pion yield of very large nova cluster ceases to be linearly proportional to the excitation mass as we assumed it to be.

Figure 9 Double rapidity distribution calculated for the reaction $\mathrm{K}^{+} \mathrm{p}\left(\mathrm{K}^{\circ}, \pi^{-}\right)$at $16 \mathrm{GeV} / \mathrm{c}$. Quantity $\mathrm{d}^{2} \sigma / \mathrm{dy}_{\pi} \mathrm{dy}_{\mathrm{K}}$ is plotted versus $\mathrm{y}_{\pi}$ for four different values of $\mathrm{y}_{\mathrm{K}}$. These distributions are presented as an illustration of the trend which should be observed, since our treatment of kaon production from a proton nova involves extra assumptions. The transverse motion of 
the nova, no longer negligible here, should also give some extra effect on the kaon rapidity distribution.

TABLE I

\begin{tabular}{|c|c|c|c|c|}
\hline & Th & $\operatorname{Exp}$ & Th & Exp \\
\hline$\left\langle\mathrm{n}^{-}\right\rangle$ & 1.35 & $1.34 \pm 0.05$ & 2.37 & $2.20 \pm 0.06$ \\
\hline$\left\langle\mathrm{n}^{-}\left(\mathrm{n}^{-}-1\right)\right\rangle$ & 1.31 & $1.27 \pm 0.05$ & 3.49 & $3.55 \pm 0.10$ \\
\hline
\end{tabular}

Caption for Table I

Average values for $\pi^{ \pm} \mathrm{p}$ at $18.5 \mathrm{GeV} / \mathrm{c}$. Data are from Ref. 9. All numbers are computed with respect to $\sigma_{\text {inel }}$. Specifically elastic two prong events are subtracted from $\sigma_{2}$ before calculation of $\left\langle\mathrm{n}^{-}\right\rangle$.

TABLE II

\begin{tabular}{|c|c|c|}
\hline $100 \mathrm{GeV} / \mathrm{c}$ & $\pi+\mathrm{p}$ & $\pi-\mathrm{p}$ \\
\hline$\left\langle\mathrm{n}^{-}\right\rangle$ & 2.31 & 3.27 \\
\hline$\left\langle\mathrm{n}^{-}\left(\mathrm{n}^{-}-1\right)\right\rangle$ & 6.86 & 11.33 \\
\hline
\end{tabular}




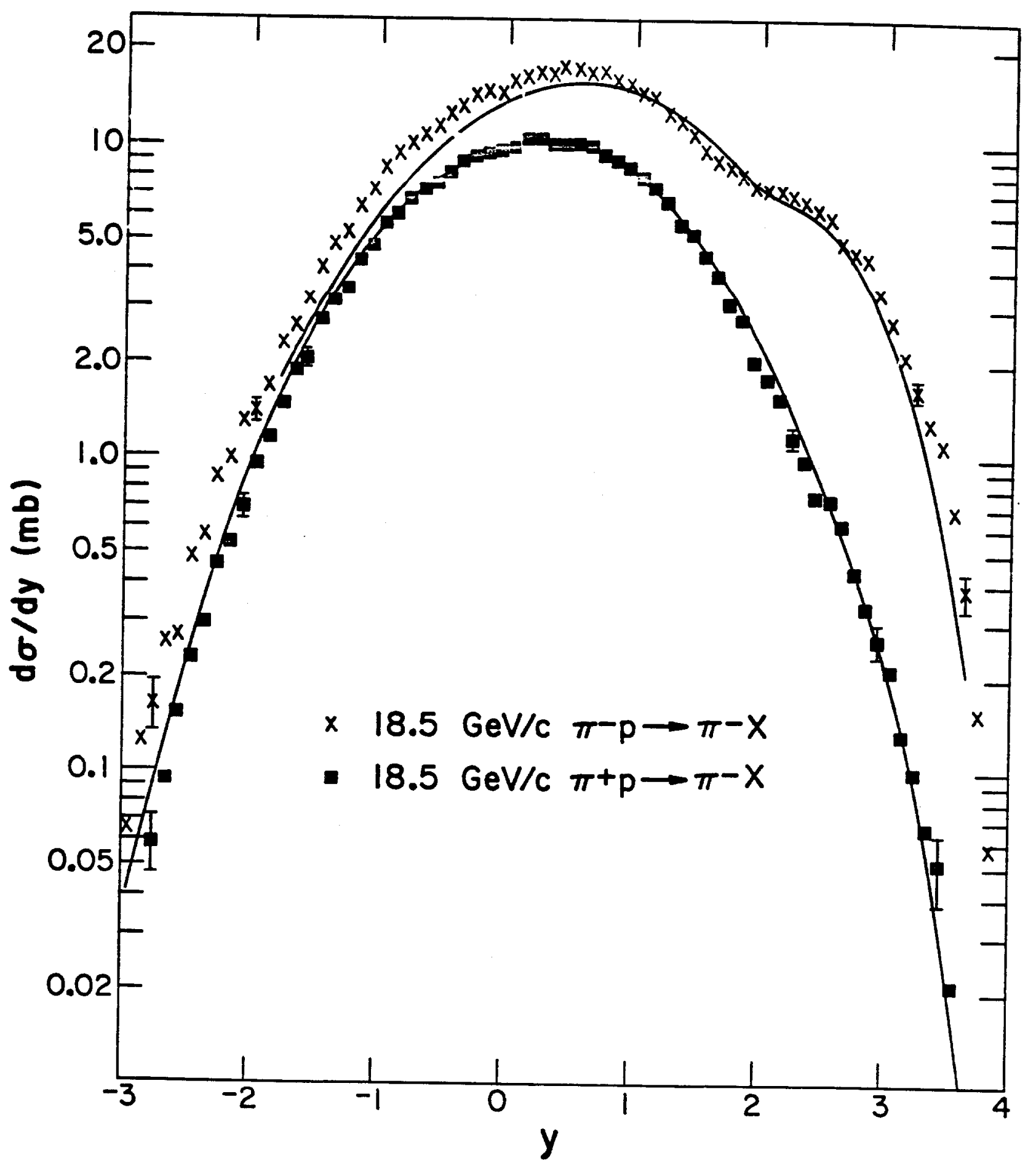


(a)

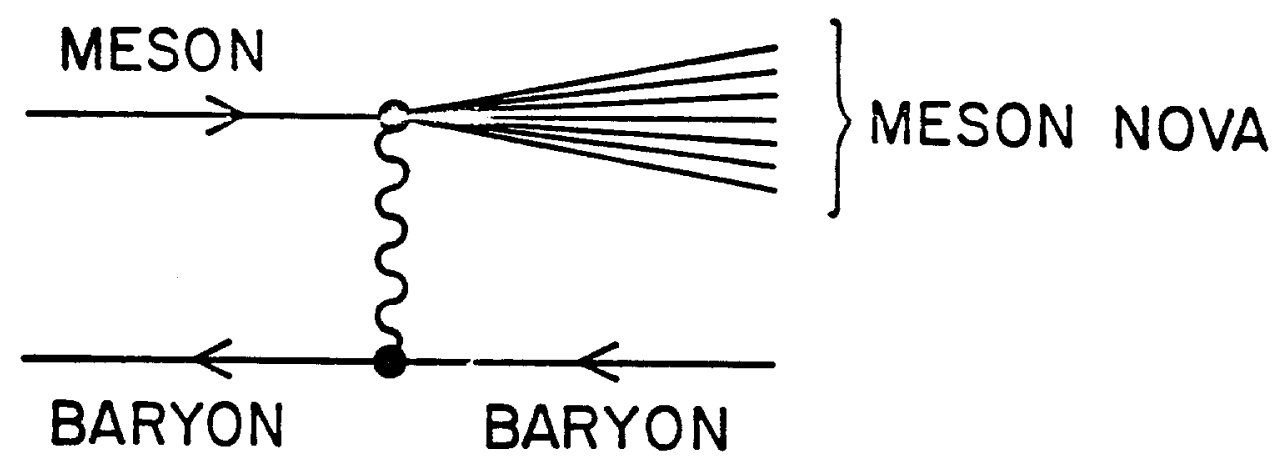

(b)
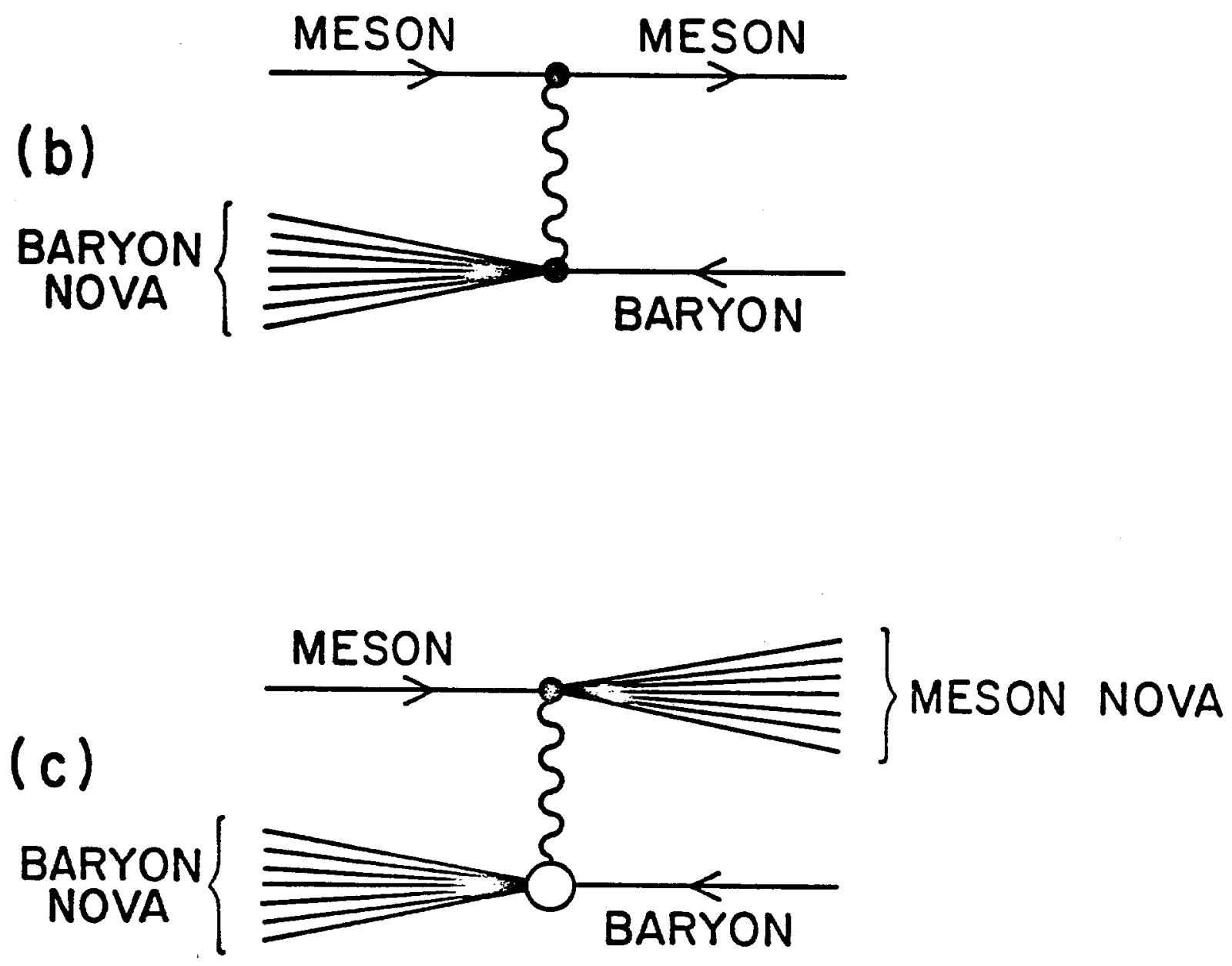


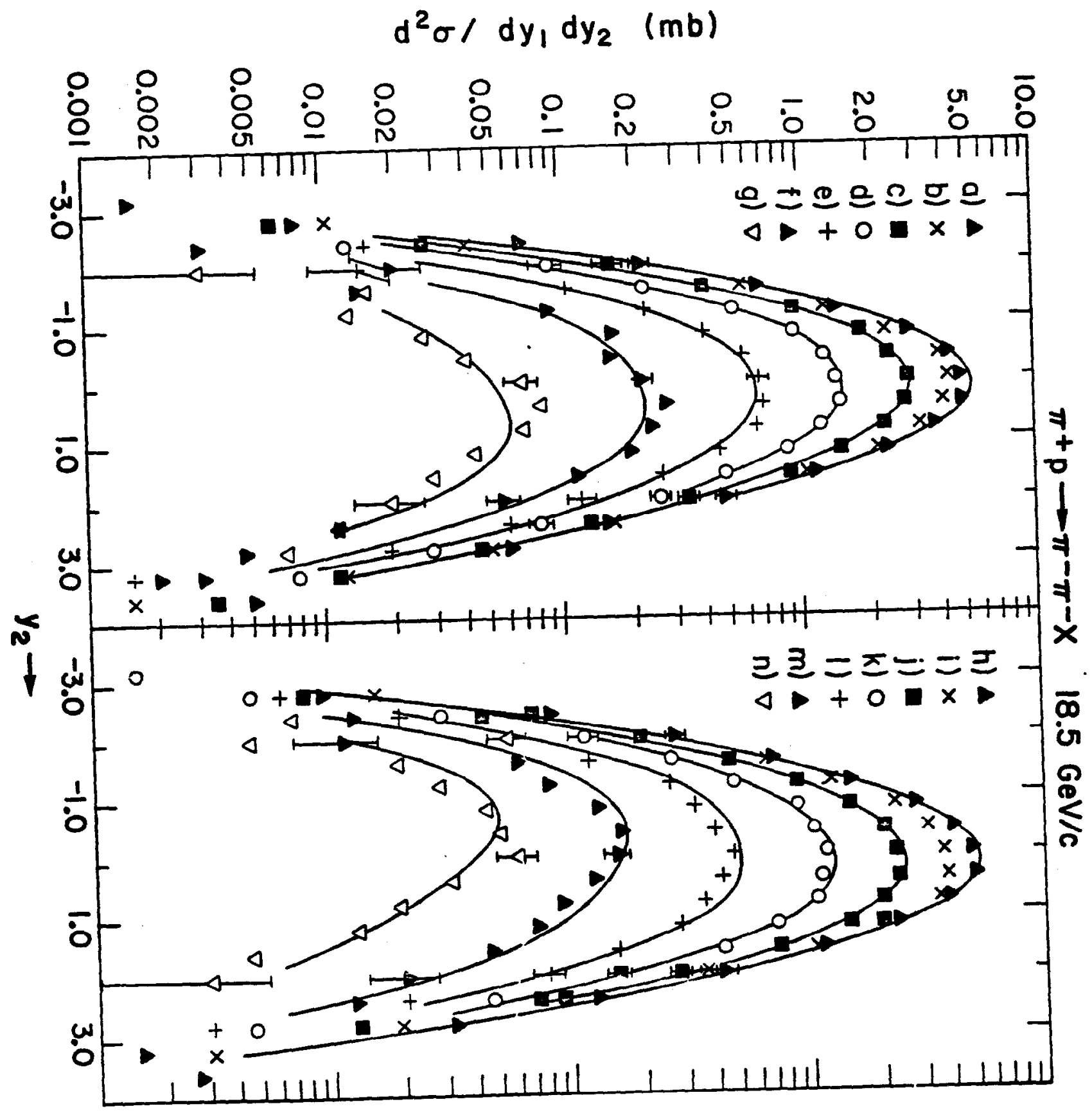




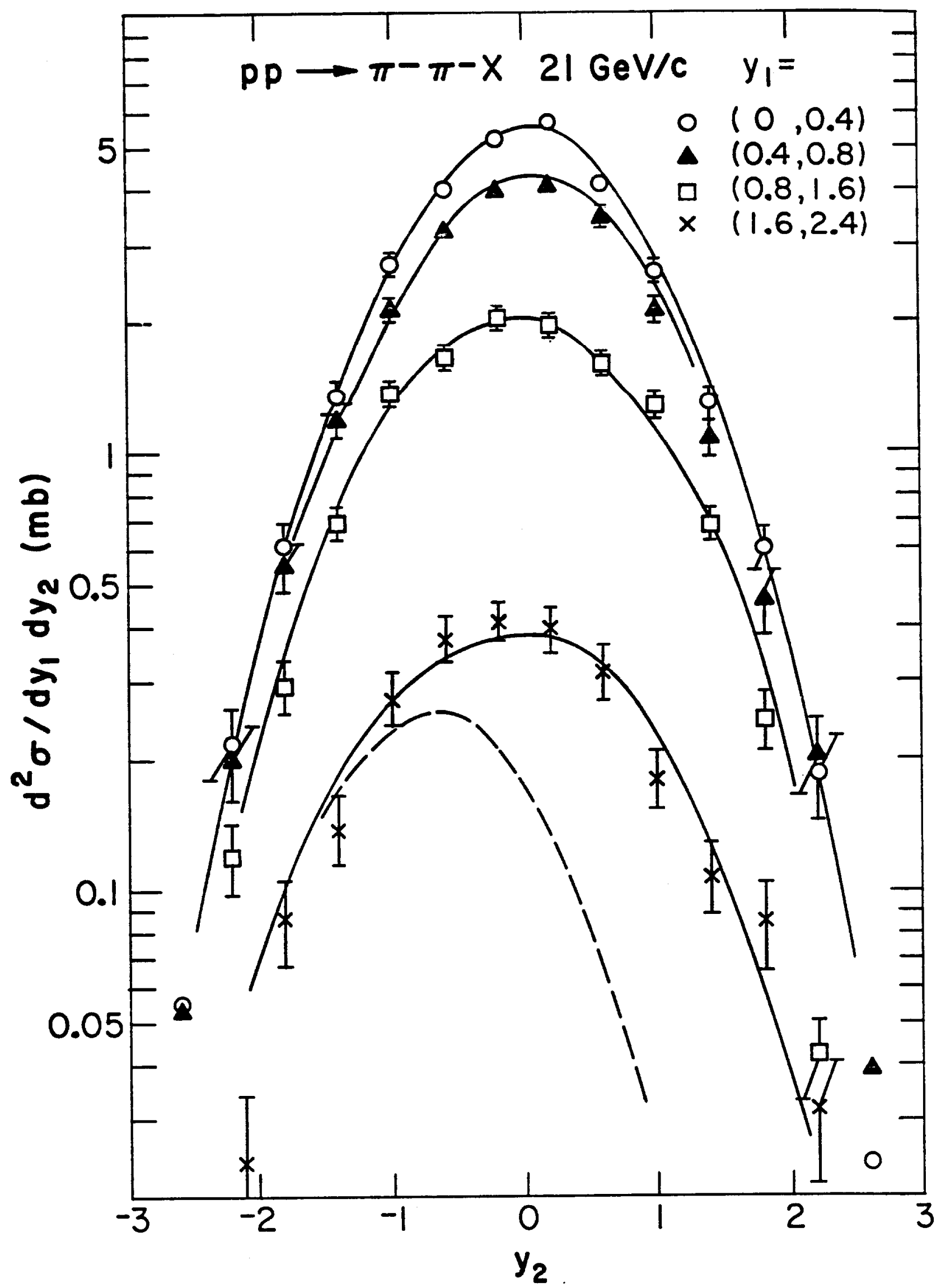




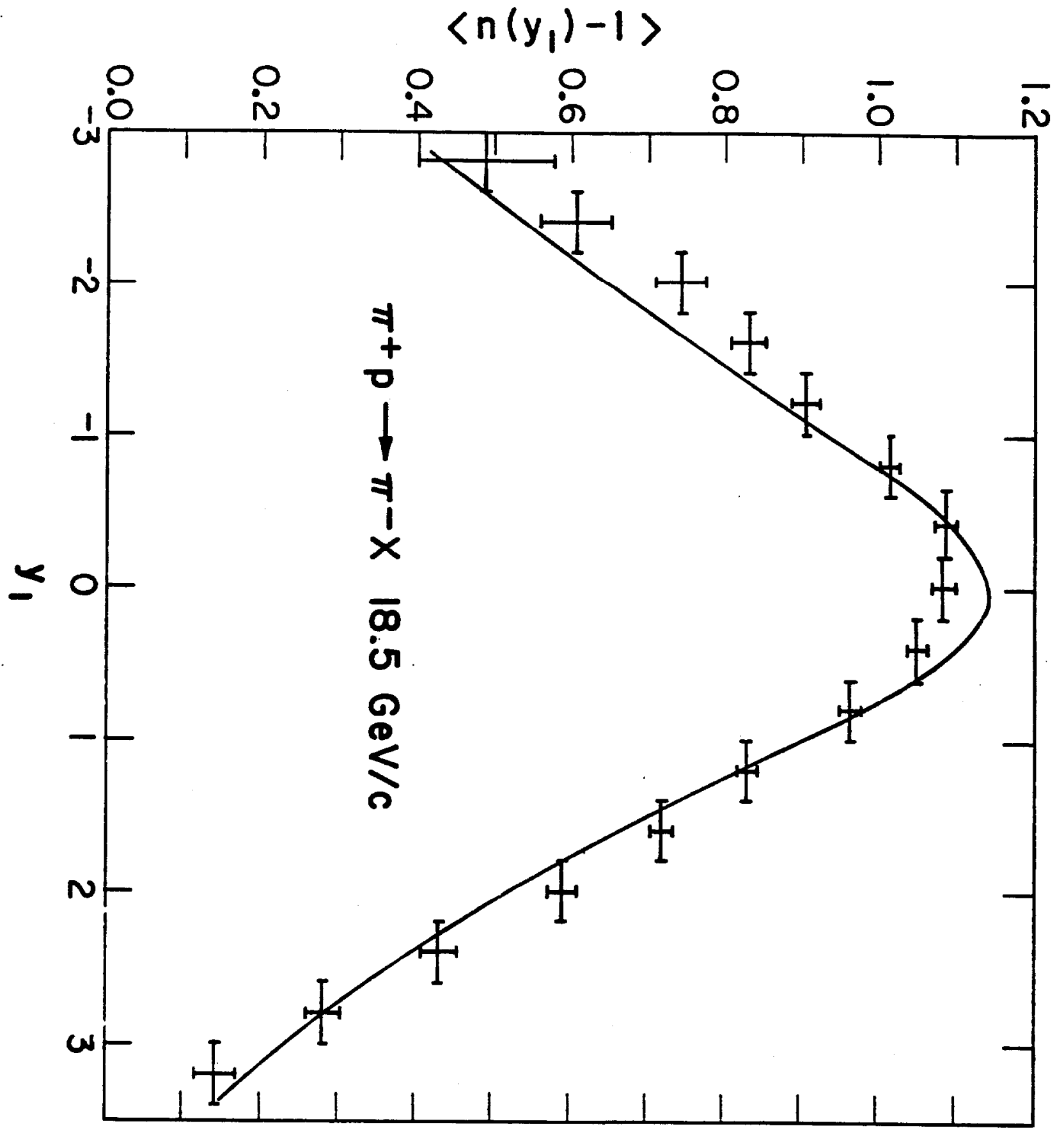




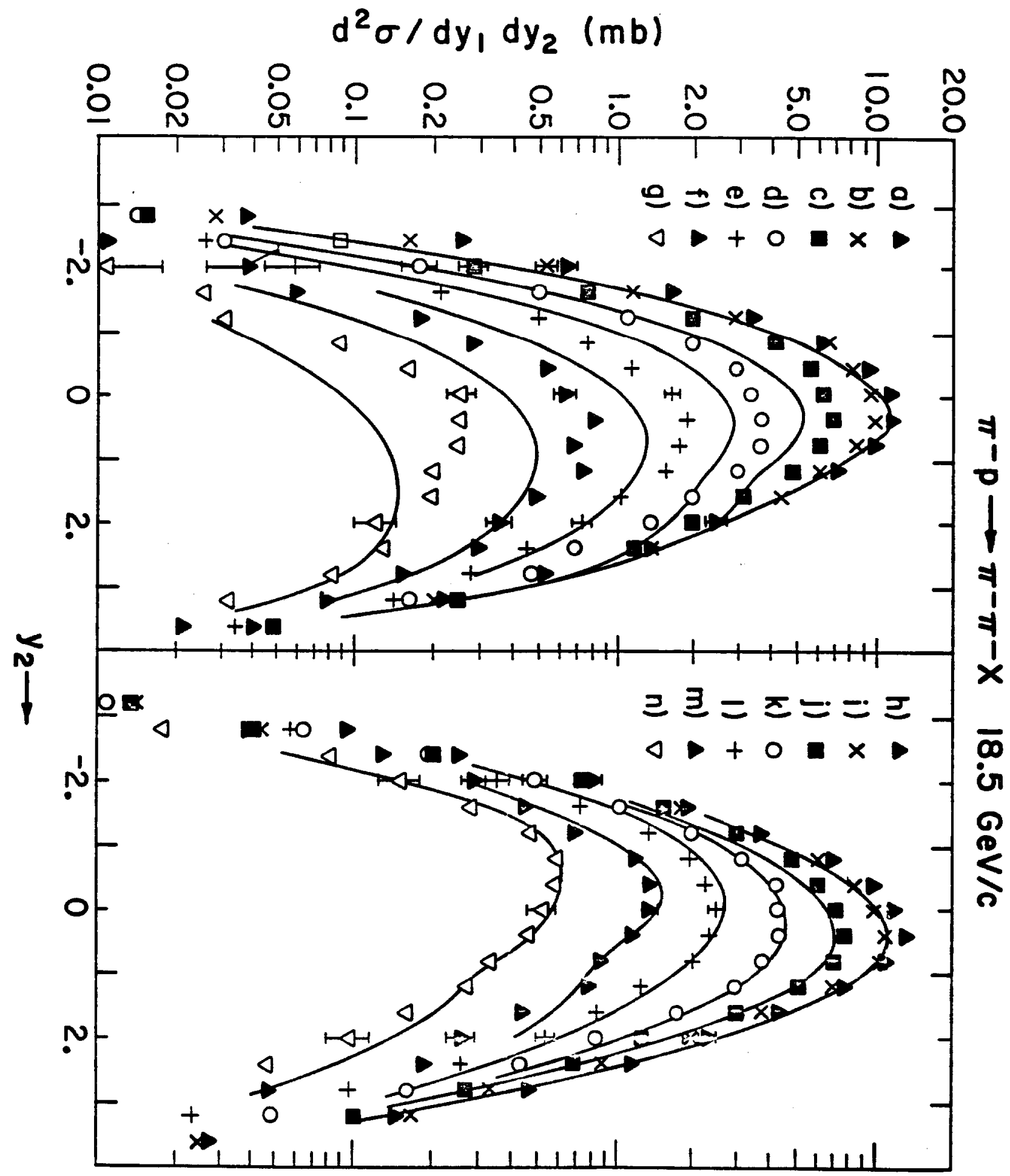




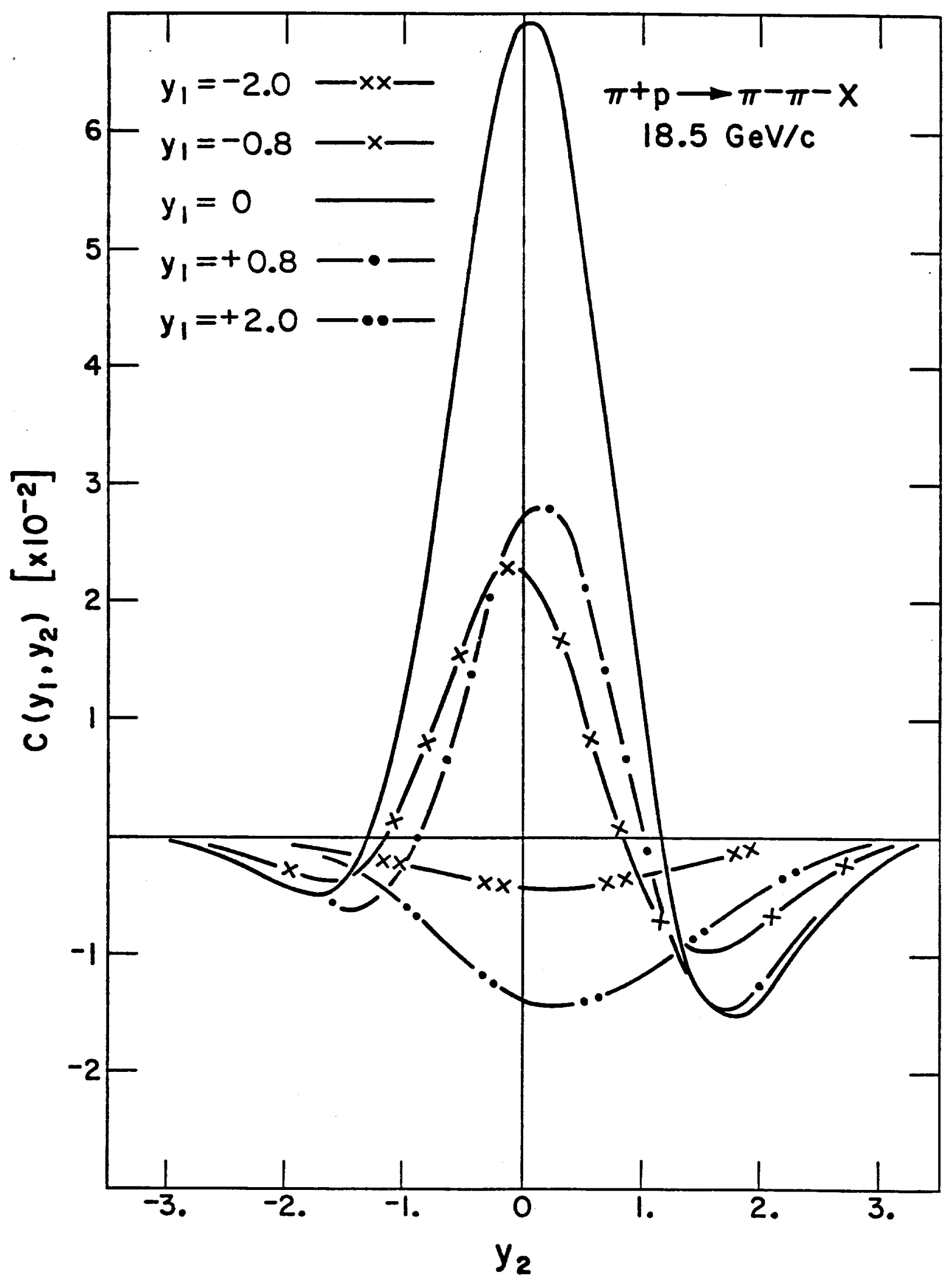




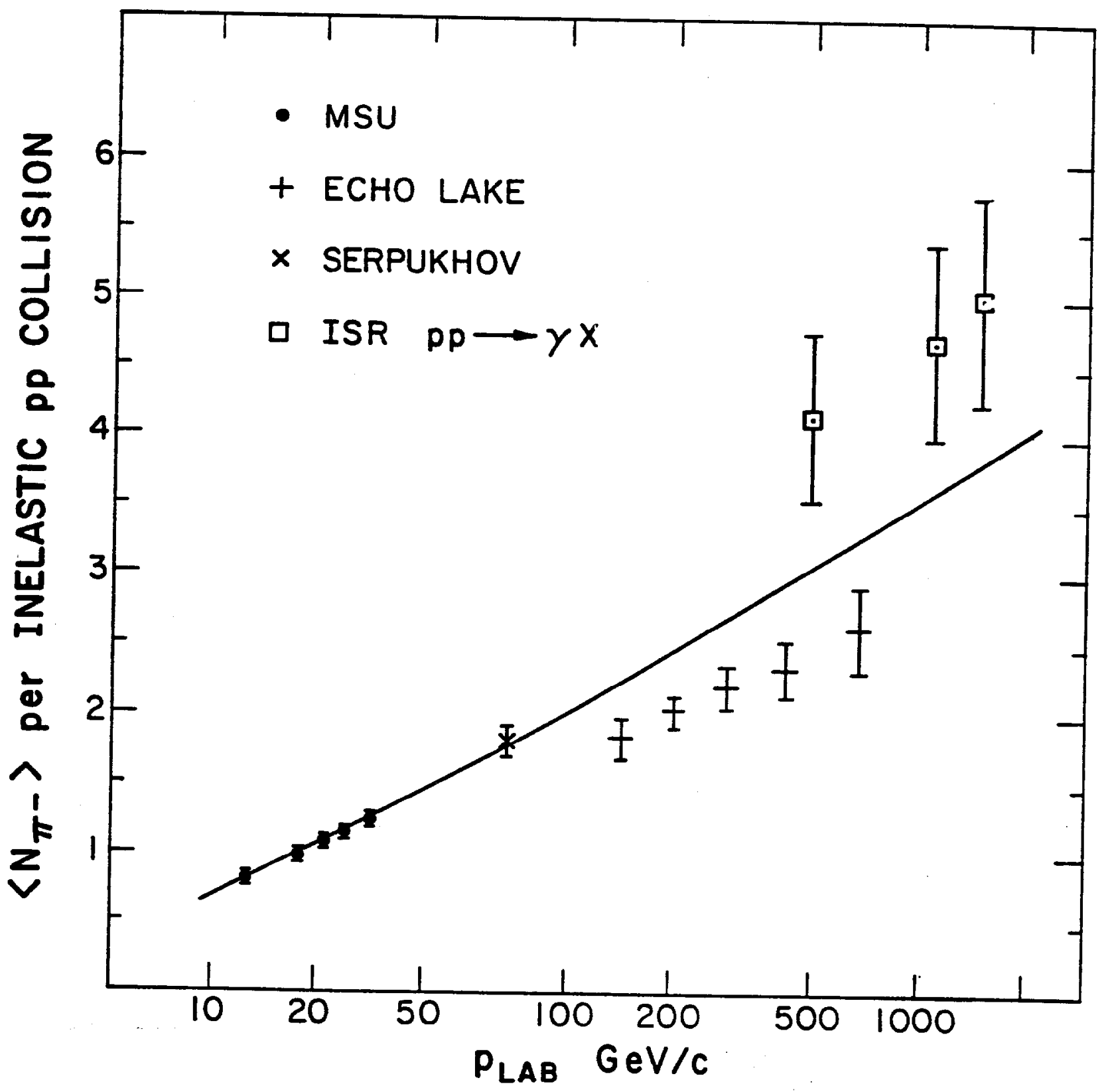




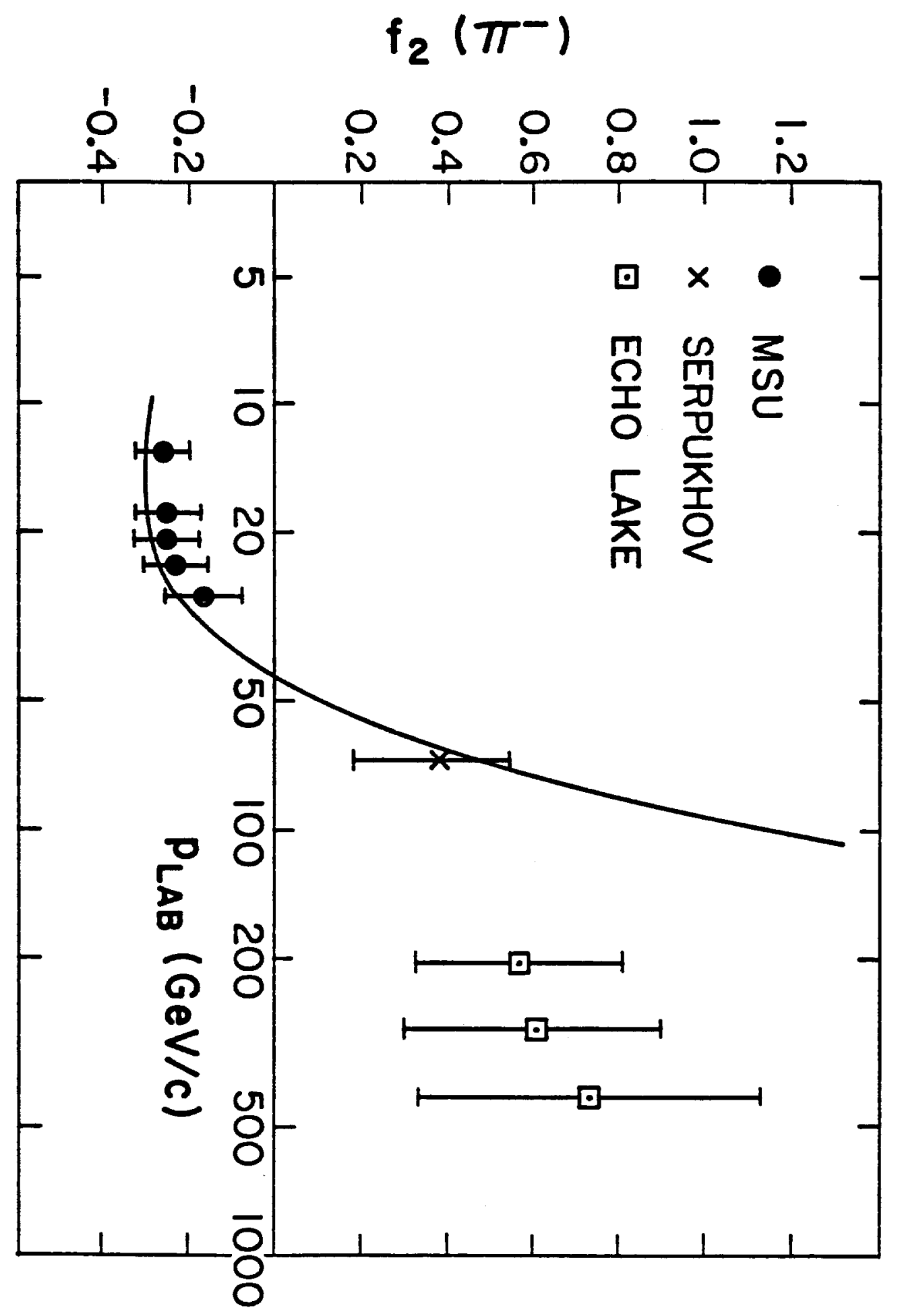




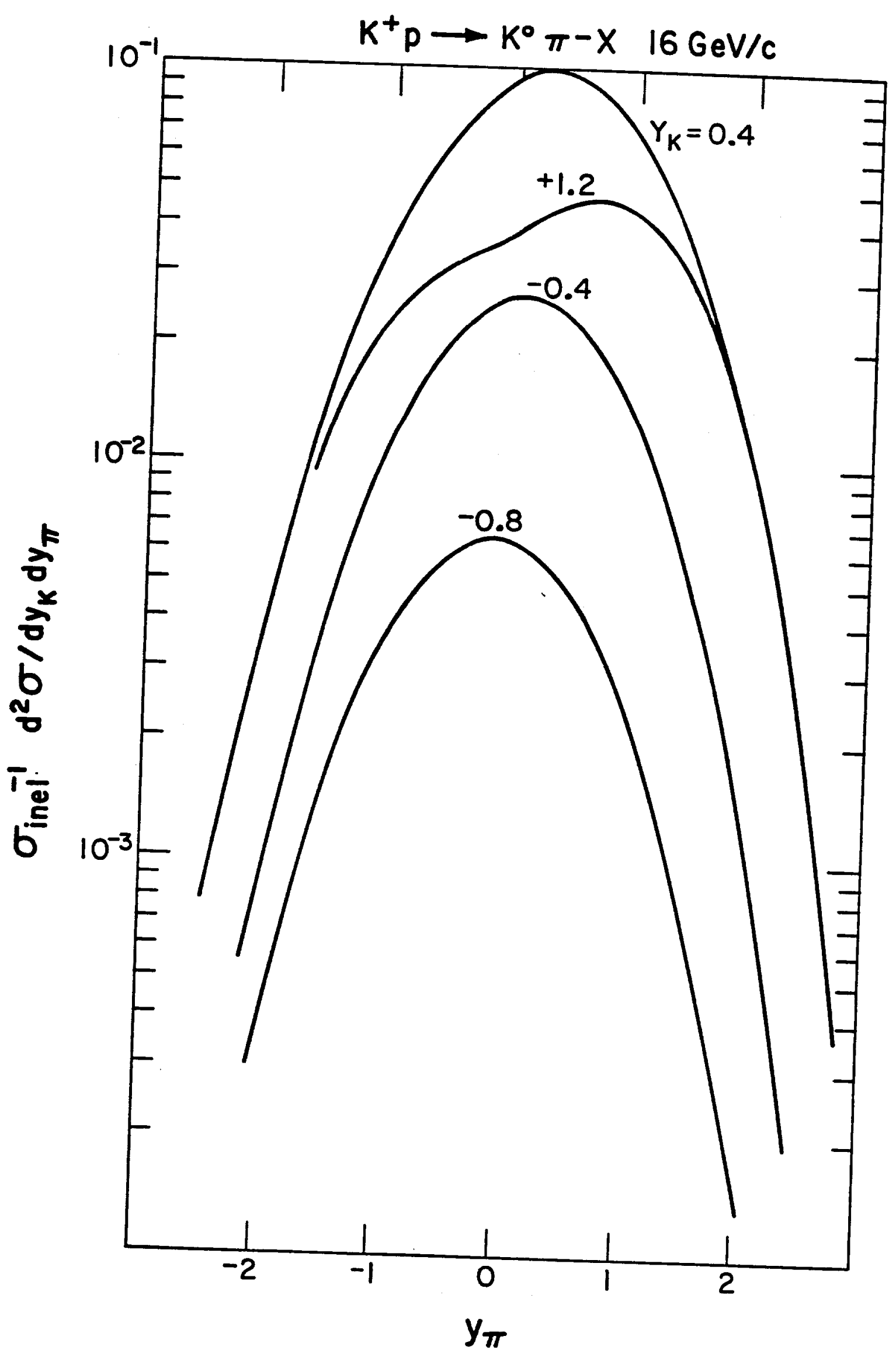

